\title{
1 Shoulder muscle recruitment during the delay period 2 encodes a reach movement plan which is sensitive to task 3 context
}

4

5 Satya Rungta ${ }^{1,2}$ and Aditya Murthy ${ }^{1}$

6

$7 \quad{ }^{1}$ Centre for Neuroscience, Indian Institute of Science, Bengaluru, India

$8 \quad{ }^{2}$ IISc Mathematics Initiative, Indian Institute of Science, Bengaluru, India

9

10 Corresponding author:

11 Prof. Aditya Murthy

12 Centre for Neuroscience,

13 Indian Institute of Science

14 Bengaluru - 576104, Karnataka, India

15 Email: adi@iisc.ac.in

16

Key words: Preparatory activity, movement initiation, isometric movements, accumulator model, motor units, high density surface EMG

Running title: Recruitment of motor activity based on task context

Number of pages: 39

Figures: 6 


\section{Abstract}

Understanding how the brain transforms motor plans into the appropriate pattern of muscle activity is a central question in motor control. Whereas some neurophysiological studies in non-human primates have shown the presence of early recruitment of motor units, other studies have reported recruitment of motor units to occur just prior to movement initiation. To address this issue in humans, and test whether early recruitment is task dependent, we studied anterior deltoid muscle activity using high density surface EMG during visually guided delayed and immediate movement tasks while subjects used either hand or isometric cursor movements. Whereas spatially specific delay period activity was present for hand movements, it was absent for isometric movements. Nonetheless, muscle activity was correlated with the time it took to initiate movements in both the task conditions. Further, we used an accumulator framework to assess initiation of movements in different task contexts. Interestingly, we found consistent but different parameters of the accumulator model to play an important role in initiating hand and cursor movements: whereas the growth rate of muscle activity was an important determinant of reaction times in delayed and immediate hand movement tasks, the onset of muscle activity recruitment played a consistent and important role in determining the reaction time for initiating cursor movements during the delayed and immediate tasks. Overall, our results reveal an unexpected heterogeneity of early recruitment as seen with high density surface EMG that may relate to the differential extent of planning engendered during actual hand movements versus isometric movements.

\section{Significance statement}

We studied the spatial and temporal aspects of response preparation in anterior deltoid muscles using high density surface EMG. Our results showed that a readiness signal could be accessed from muscle activity but were absent during isometric cursor movements. Using an accumulator framework, we show that growth rate and onset of motor unit recruitment systematically explained variability in time to movement onset based on task context. 


\section{Introduction}

58 The coupling of neural activity to muscle activity enables the transformation of motor

59 plans to movement execution. To enable tight control over such coupling, the nervous system

60 has evolved multiple possible mechanisms to gate the flow of information between the

61 central nervous system and the peripheral musculature that involves the motor cortex

62 (Kaufman et al. 2014), the basal ganglia (Alexander et al. 1991; Graybiel 1990; Hikosaka and

63 Wurtz 1983a, 1983b; Mink 1996; Parent and Hazrati 1995), as well as the brainstem and

64 spinal cord circuitry (Cohen et al. 2010; Prut and Fetz 1999; Selen et al. 2012; Sinclair and

65 Hammond 2009; Zinger et al. 2013). However, despite the presence of gating mechanisms,

66 certain behavioral paradigms that typically evoke reflexive activity can initiate reflexive

67 recruitment of proximal limb muscles in cats, monkeys and humans (Fautrelle et al. 2010;

68 Perfiliev et al. 2010; Saijo et al. 2005; Schepens and Drew 2003), and stimulus-linked

69 activity can also be recorded from shoulder muscles during such rapid reflexive movements

70 but not during delayed movements (Pruszynski et al. 2010), suggesting that muscle activity is

71 gated by inhibitory spinal mechanisms for deliberative movements that require voluntary

72 preparation (Georgopoulos et al. 1986; Kettner et al. 1988; Lecas et al. 1986; Requin et al.

73 1990; Tanji et al. 1988).

The notion that inhibitory processes in the spinal cord are activated during the preparatory period is also consistent with previous surface EMG studies showing a systematic decrease in the amplitude of monosynaptic reflexes; and is also observed as a decrease in motor potentials evoked by TMS when the duration of the delay remained constant throughout a block of trials (Bonnet et al. 1981; Brunia 1983; Burle et al. 2004; Davranche et al. 2007; Hasbroucq et al. 1999; Touge et al. 1998). Consistent with this view Duclos et al. 2008 have also observed decreased activity in motor unit activity. More recently, such preparatory induced changes have also been observed in the spindle afferents of antagonistic muscles (Papaioannou and Dimitriou 2021), which is thought to reflect the setting up of a "fusimotor set" prior to movement initiation (Prochazka et al. 1985). These results are consistent with the notion that that the observed inhibitory gating in the spinal cord during the preparation of movement may primarily serve to decrease the reflex gains in anticipation of voluntary movement without necessarily affecting the initiation times of voluntary movements. 
In addition to suppressing reflex gains, delay period activity that are routinely observed in motor cortical cells (Requin et al. 1990; Tanji et al. 1988), as well as in oculomotor areas such as the Frontal Eye Fields (FEF) and the Superior Colliculus (SC) (Dorris and Munoz 1998; Rungta et al. 2021), are also correlated with benefits in reaction time, raising the possibility that central preparatory activity may have also direct facilitatory effects on agonist muscle activity as well, possibly via more direct connections from the reticulospinal tracts on spinal/cervical motor neurons (Kearsley et al. 2021). Consistent with this notion, Mellah et al. 1990 have shown the presence of delay period motor unit activity in axial and proximal skeletal muscles during a delayed movement task in monkeys. More recently, Rungta et al. 2021 have also observed the presence of delay period activity in neck muscle activity during a delayed saccade task in head fixed monkeys, confirming previous reports of anticipatory preparatory activity from surface EMG recordings from the neck splenius capitis muscle of monkeys (Roesch and Olson 2003). Interestingly, Rungta et al. 2021 observed a heterogeneity of responses amongst motor units, such that only units with smaller amplitudes showed a clear modulation during the delay period. Further, the observed ramping activity was modelled using an accumulate to threshold model (Asrress and Carpenter 2001; Carpenter and Williams 1995; Hanes and Schall 1996; Nelson et al. 2016) to provide a link between the observed motor activity and saccade initiation times. Thus, taken together this line of evidence suggests the possibility that some motor unit activity may reflect a more direct readout of central activity of motor preparation and planning.

To test these ideas further, we revisited the question of whether the delay period activity can be elicited from arm muscles during reaching movement in humans analogous to what was observed from the neck muscles in monkeys during a delayed saccade task. Motivated by the notion that early muscle activity maybe more influenced by smaller motor units that are activated during the preparatory period, we used high density surface electromyography to assess spatially localized muscle activity, that may emphasize the contribution of smaller motor units that would otherwise be obscured by standard whole muscle EMG activity (Gazzoni et al. 2004, 2004; Merletti and Farina 2009; Rau and Disselhorst-Klug 1997). In the process, we also tested whether such delay period activity was sensitive to task context, which could explain the divergent observations regarding the presence or absence of early motor unit activity during the planning of reach movements in humans. Finally, we tested whether an accumulation to threshold model, that has provided a simple framework to understand the initiation of saccadic movements can be used as a 
121 linking hypothesis to test the relation between the motor unit activity and the initiation of reach movements and their dependence on task context.

\section{Materials and methods}

\section{Subjects}

For this study, we recorded from 9 and 11 male subjects (age group: 26-32) for the hand and cursor movement tasks, respectively. Prior to the experiments, the subjects had to fill the consent form in accordance with the approved protocol of the Institutional Human Ethics Committee, Indian Institute of Science, Bangalore. Subjects were paid for their participation in the experiments based on the number of correct trials they performed.

\section{Task and stimuli}

Visually-guided delayed and immediate movements

Subjects were instructed to make movements to the target location as smoothly as possible, without making abrupt velocity changes. After fixation $(300 \pm 15 \mathrm{~ms})$ on a central box (FP: white, $0.6^{\circ} \times 0.6^{\circ}$ ), a stimulus (Target: green, $1^{\circ} \times 1^{\circ}$ ) appeared at the periphery of the screen. The target could appear at any one of the eight locations lying with an eccentricity of $12^{\circ}$ from the fixation box in the cursor movement task. For the hand movement experiments, to maximise the number of trials, the target location was restricted to only 2 positions, i.e., $90^{\circ}$ or $135^{\circ}$, towards the movement field, and diagonally opposite locations, $270^{\circ}$ or $315^{\circ}$, away from the movement field. Subjects had to maintain fixation until the central box disappeared. The disappearance of the box was a go 'cue' to make a hand or cursor movement to the target location. The time interval or the delay period (hand: 0ms and 1000 $\pm 150 \mathrm{~ms}$; cursor: $0 \mathrm{~ms}$ and $750 \pm 150 \mathrm{~ms}$ ) between the go cue and the appearance of the target, separated 'where' from 'when' to initiate a movement. After a successful trial, the subjects heard a 'beep' sound, as a reward, indicating the trial was carried out correctly. An electronic window of $\pm 2^{\circ}$ drawn from the centre of the fixation box and the target were used to assess the online performance of the subjects. Subjects were rewarded for each correct trial. 


\section{Experimental setup}

151 The setup used for carrying out experiments for hand and cursor movements are 152 described below:

\section{Hand movements}

154 All the experiments in which subjects had to reach out or make an actual hand 155 movement were conducted in a dark room. A sensor along with an LED was strapped on the 156 pointing finger to record hand movements and provide visual feedback. Stimuli were 157 displayed using a 24" LED DELL monitor $(60 \mathrm{~Hz})$, placed face down on a wooden setup as 158 shown in Fig S1 (https://doi.org/10.5281/zenodo.6026951). A semi-transparent mirror (25\% 159 transmission, $75 \%$ reflectance) was placed at an angle below the monitor screen to reflect the 160 stimuli onto an acrylic sheet placed parallel to and below the mirror. The setup was necessary 161 so that the monitor could not distort the electromagnetic field used for detecting hand 162 movements. The subjects could use the chin rest and found no difficulty in doing the 163 experiments.

\section{Cursor movements}

The force applied by the subjects on the robotic arm (Kinarm end-point robot, BKIN 166 Technologies Ltd, Canada), was mapped on to cursor movements displayed on a 24"' LED 167 DELL monitor, placed in front of their experimental setup. Subjects were instructed to apply 168 force using their shoulders and not to make any sort of arm and hand movements.

\section{Data collection}

Experiments were carried out using a TEMPO/VIDEOSYNC system (Reflecting

171 Computing, USA). The codes for generating stimuli were written and controlled using

172 TEMPO. The visual stimuli were displayed using VIDEOSYNC software on a Sony Bravia 173 LCD monitor (42 inches, $60 \mathrm{~Hz}$ refresh rate; $640 \times 480$ resolution) placed $57 \mathrm{~cm}$ from the 174 subjects, so that $1^{0}$ of visual angle corresponds to $1 \mathrm{~cm}$ on the screen. While subjects were 175 performing the task, we monitored their hand position and recorded electromyographic 176 signals from the anterior deltoid shoulder muscles. 


\section{Hand tracking system}

178

179

180

181

182

An electromagnetic tracker (240 Hz, LIBERTY, Polhemus, USA) was placed on the tip of the pointing finger. The position of the sensor with reference to a source was read out by the system and sent to the TEMPO software in real time. At the beginning of the session, the experimenter would ensure that hand movements were being made to the correct target location and the traces landed within the electronic window (visible only to experimenter) which was centred on the target. The fixation spot and the horizontal and vertical gains of the hand data were fixed as constant values based on calibration of the system.

\section{Mapping force to cursor movements}

Cursor movements were generated by having subjects make isometric hand movements using a Kinarm end point robot (BKIN Technologies Ltd, Canada). Cursor movements were mapped on to three different force levels by changing the gains of the data obtained from the force sensor placed on the handle that was locked in a central position (see Fig S2, https://doi.org/10.5281/zenodo.6026951). For each trial, the colour of the fixation box (colours: red, yellow or green) indicated the force (levels: high, intermediate or low) needed to be applied by the subject to move the cursor from the fixation box to the target in the absence of an overt hand movement (isometric condition).

\section{Surface electromyography recordings}

EMG activity was measured using a high-density electrode array built by joining three 10 pin Relimate connectors. Out of multiple electrodes, an eight 8-channel array setup (see schematic, Fig 1C) was used for recording. The data was sampled at $10 \mathrm{kHz}$ and band-passed between 10-550 Hz and stored in the Cerebrus data acquisition system (Blackrock Microsystems, USA). The reference electrode was also placed within the array, while a ground electrode was placed on or at the back of the ear lobe of the subject. 


\section{Data Analysis}

\section{Calibration of the hand tracker}

The average accuracy across different sessions for the hand tracker, while fixating was around $0.0035 \pm 0.0001 \mathrm{~cm}$. This was estimated using the jitter while fixating prior to the appearance of the target. Also, the average spatial end point and accuracy of the hand movements to the target locations was $11.32 \pm 0.01 \mathrm{~cm}$. Accuracy was estimated by measuring the mean hand end-point location during a $150 \mathrm{~ms}$ period of the post hand fixation time across different sessions.

\section{Calibration of force for cursor movements}

The robotic arm was locked at the centre position using a heavy triangular base device. Initially, when no force was applied on the manipulandum of the robotic arm, the position of the cursor was set to the centre of the screen. Vertically hanging weights were used to apply horizontal forces on the robotic manipulandum using a custom-built pulleybased system. Displacement of the cursor from the centre position was recorded by varying the forces applied on the robotic arm to calibrate the system. Three different gains were used to map three different force levels onto the same cursor displacements during the cursor movement task (see Fig S2, https://doi.org/10.5281/zenodo.6026951). The average accuracy across different sessions for the cursor, while fixating was around $0.19 \pm 0.0082 \mathrm{~cm}$. This was estimated using the jitter while fixating prior to the appearance of the target. Also, the average spatial end point accuracy of the cursor movements to the target locations was 11.91 $\pm 0.013 \mathrm{~cm}$. Accuracy was estimated by measuring the mean cursor end-point position during a $150 \mathrm{~ms}$ period of the post cursor fixation time across different sessions.

\section{Hand and cursor onset detection}

Hand and cursor onsets were detected offline. The noise level in the distance trace profile captured during the fixation time was used as a criterion to set a threshold to mark the beginning of hand movement. Multiple check points were incorporated in the algorithm to ensure reliable hand detection. Firstly, the cutoff criterion was set at a threshold of 2.5 standard deviations above the average noise level observed in the sensor data during the fixation time for each trial. Secondly, the onset point from where the hand traces were seen as increasing monotonically for more than $60 \mathrm{~ms}$ (15 bins) was marked as the time for the 
235

236

237

238

239

beginning of the hand movement. Thirdly, trials in which the hand latency was less than 100 ms or more than $1200 \mathrm{~ms}$ were rejected as anticipatory or delayed movements.

\section{Extraction of motor units}

Surface electromyographic (sEMG) recordings were analysed in Matlab (Mathworks, R2018b). A Threshold criterion was used to extract units from the data set. The criterion (2.25 SD) was set based on the initial $500 \mathrm{~ms}$ of the data from the beginning of each trial. The procedure was carried out by initially using EMGLAB (McGill et al. 2005) software. In short, the software collected the template of the waveforms (with window length of $20.33 \mathrm{~ms}$ for motor units) and the time stamps for all the local maxima that were above the threshold. Next, principal component analysis and template matching were used for sorting spikes. Initially, 3 to 4 templates were isolated for each trial using principal component analysis. All the templates were then collected (typically $>\sim 600$, each session) and based on these templates, further sorting was done. The algorithm once again performed PCA to isolate units ( $2-3$, isolated by the experimenter) which were found to be stable throughout the session. Finally, based on minimum least square distance value between each waveform and different templates, units were sorted into appropriate groups. All the units that were extracted were then combined to obtain multi-unit motor activity (MUA) which was used for further analysis.

\section{Exclusion of units}

Units were discarded if they could not be isolated clearly or if they did not show taskrelated response. Units which were only, bi and tri-phasic were considered for analysis. To remove motion artifacts during sessions, units which were saturated or with amplitude greater than $\pm 5000 \mu \mathrm{V}$ were not considered for analysis.

\section{Spike density function (SDF)}

A spike density function was used to characterize the activity pattern of motor units. For each trial, spike trains were aligned with respect to the time when an event (example: onset of a movement) occurred. The mean activity pattern of the motor units could be 
264 estimated by averaging the convolved data over multiple trials. The spike trains were

265 convolved in both forward and backward directions using a gaussian filter with a std.

266 deviation of $10 \mathrm{~ms}$. One advantage of processing signals using the above method is that all

267 the features in the filtered data are exactly where they occurred in the unfiltered format.

268 Henceforth, in the results the SDF is referred to as motor activity.

269

270

271

272

273

\section{Movement fields}

The response or the movement field for the anterior deltoid muscle was defined for all sessions showing movement direction selectivity prior to the onset of a movement (see Fig $\underline{\mathbf{S 4}}$, https://doi.org/10.5281/zenodo.6026951). For hand movements, before the session began, subjects were instructed to reach for targets shown in a sequence across the screen in a $(4 \times 4)$ grid. These targets covered the workspace for hand movements. While the targets were shown, EMG signals recorded from this workspace was used to map the movement field (MF) as described in equation 1 below (see Fig S1 for representative hand session). Once, mapped only 2 target positions, in and out of MF were shown to subjects to maximise number of trials in a session. For cursor studies, out of the eight target locations, the four positions which showed higher activity during the movement epoch were grouped as inside movement field (in-MF) and the diagonally opposite positions were grouped as outside movement field (out-MF). Since, the movement field for muscle activity were large, we combined the results from four different target positions to leverage higher statistical power. A cosine tuning function was used to identify the preferred direction for each session. The target locations which lied within $\pm 90^{\circ}$ of the preferred direction was grouped as in-MF. The activity during the movement epoch for all correct trials was regressed with the direction of the target according to equation 1, using the Statistical toolbox in MATLAB.

$$
\hat{Y}(\theta)=A \cos \left(\theta+\theta_{P D}\right)+B
$$

Here, $\widehat{Y}(\theta)$ represents the motor activity when the target was presented at an angle $\theta^{0}$, $\mathrm{B}$ is the baseline firing activity of the shoulder muscles and $\theta_{\mathrm{PD}}$ is the preferred direction for the multi-unit motor activity (MUA). 


\section{Methods for analysing EMG data:}

293 We used two methods to analyse EMG data. First, we used a standard method such as 294 the root mean square of the EMG signal to analyse the recruitment of anterior deltoid 295 (shoulder) muscles. Mathematically, the root mean square measures the variability present in 296 the signal and is thought to reflect information about the recruitment of motor units. A running window of length $35 \mathrm{~ms}$ with steps of $1 \mathrm{~ms}$ was convolved with the square of the data. The square root of the convolved data for each trial was then used for further analysis. Additionally, we isolated spike waveforms from the EMG signal and extracted their time of occurrence and constructed a spike density function from this data. Both these approaches led to comparable results (Fig 1; Pearson's r: 0.73; $\mathrm{p}<0.001$ ).

Furthermore, we used a standard 'Additive time series decomposition model' to split the motor activity into its underlying components, namely - trend, cycles and residuals.

$$
\operatorname{Activity}(t)=\operatorname{Trend}(t)+\operatorname{Cycles}(t)+\operatorname{Residuals}(t)
$$

A moving average using a window of 30 bins or $150 \mathrm{~ms}$ was used to estimate the trend component present in the time series data. A maximum of 2 to 3 peak cyclic components were identified as cyclical patterns and the remaining irregularity components were verified to be residuals or as white noise after using an auto regressive and moving average (ARMA) approach. For this paper, we focus our analysis and discussions on underlying trend patterns for the time series data only.

\section{Direction discrimination time (DDT)}

A standard receiver operator characteristic (ROC) analysis was performed on SDF or motor activity for each time bin after the response (MF-in and MF-out) were aligned on the go cue. The area under an ROC curve ranges from 0.5 to 1 . For each time bin, an ROC value of 0.5 would imply that the motor activity could not be reliably distinguished between in and out of the movement field, whereas an ROC value of 1 would mean complete separation between the two distributions. A threshold criterion during reaction time was set at an ROC of 0.7. The time at which the ROC value crossed the threshold for a continuous period of 150 ms was marked as the direction discrimination time (DDT). Since the ROC time series had cyclic components, it was smoothened using a uniform filter of $45 \mathrm{~ms}$ with zero phase shift 
and then minima between a period of $850 \mathrm{~ms}$ prior to go cue and $25 \mathrm{~ms}$ after go cue was chosen as the starting point to demarcate when the ROC trend increased monotonically during the delay period.

\section{Categorisation of trials based on different parameters}

Reaction time was used as the metric for assessing the initiation of movements. For each session, the data was divided into 2 different groups. Based on this division, different trials were categorised into different conditions - slow and fast reaction times. Trials with RT less than 45th percentile were classified as fast RT trials, whereas trials above the 55th percentile onwards were grouped into slow RT trials.

\section{Components of the accumulator model}

Next, we wanted to test whether a linear accumulator model (Carpenter and Williams 1995; Noorani and Carpenter 2016; Reddi and Carpenter 2000) could be extended to the periphery to study initiation of movements. Various components of this framework were measured for each session based on their spike density function, under MF-in conditions for slow and fast reaction times. Baseline activity, activity at go cue and activity at movement initiation or threshold activity were measured by averaging the spiking activity of each session prior to the target, go cue and movement onset, respectively. Different approaches were used to estimate the slope and onsets for capturing the trend component for each session during reaction time. First, a fixed interval from $50 \mathrm{~ms}$ prior to the go cue upto $500 \mathrm{~ms}$ following the go cue, was used to estimate the average slope using a simple linear regression approach. The time at which the global minima occurred over the same interval was considered as the onset value. Second, a piecewise simple regression was carried out using a $175 \mathrm{~ms}$ moving window during the delay period to estimate the growth rate based on different metrics respectively- (a) maximum slope (b) best linear fit ( $\mathrm{r}$ square). Under both the conditions, the value of the slope obtained at the global maxima index from the time series obtained after applying the moving window was considered as an estimate for the slope value. The estimated slope was then used to obtain the intercept on the $\mathrm{x}$ axis. Further, to be more conservative in our approach, the onset value was re-estimated to obtain the time at 
352 which the global minima occurred over the interval between and from the intercept value up

353 to the go cue. Third, a closing window approach with a rightward shift of $1 \mathrm{~ms}$ with each bin 354 from $50 \mathrm{~ms}$ prior to go cue upto $500 \mathrm{~ms}$ after the go cue during reaction time was used to 355 estimate the growth rate using linear regression. As described above in approach two, we 356 used the slope value obtained at the global maxima index from the time series obtained in the 357 previous step; to get an estimate for the slope value for both the metrics respectively. Similar 358 to approach 2, the onset value was estimated to obtain the time at which the global minima 359 occurred over the interval between and from the slope intercept on the $\mathrm{x}$ axis up to the go cue. 360 Since the second method gave us the most consistent results, we used this method for all the analyses pertaining to Figs 5 and 6.

\section{Statistical tests}

All statistical tests were done on population data across subjects (unless specified). A 364 Lilliefors test was carried out for checking normality of the data, under each condition. A Nway ANOVA was carried out on data when necessary, where $\mathrm{n}$ represents the number of multiple factors. A standard t-test or paired t test was carried out whenever required. If normality failed, a sign test was done instead of paired sample t-test, and a Kruskal Wallis test was done for ANOVA. A Binomial test was done to test whether the trend in the data was above chance level. In-built Matlab functions and the statistical toolbox were used to carry out appropriate analysis. 


\section{RESULTS}

374 Subjects made delayed and immediate hand or cursor movements (Fig 1A). Targets could appear in any one of the eight locations, followed by the disappearance of the fixation box, which marked the go cue to initiate delayed or immediate movements to the required target location. The delay period allowed for successful encoding of the target stimulus and could be used to prepare for an upcoming movement. We recorded from 20 subjects with 9 and 11 sessions for hand and cursor movements. Significantly shorter reaction times (see Fig 1B) were observed for delayed movements when compared to immediate movements for both hand (delayed task: $397.14 \pm 36.63 \mathrm{~ms}$ and immediate: $511.13 \pm 35.24 \mathrm{~ms} ; \mathrm{t}(8)=7.28, \mathrm{p}<0.001$ ) and cursor studies (delayed task: $377.83 \pm 11.04 \mathrm{~ms}$ and immediate: $492.62 \pm 27.51 \mathrm{~ms} ; \mathrm{t}(10)=$ $6.17, \mathrm{p}<0.001)$. These results indicate that some aspect of motor preparation occurred during the delay period which conferred a reaction time advantage.

An eight (8) channel high-density electrode array setup (for schematic, see Fig 1C) was used for recording electromyographic (EMG) signals. Shown in Fig 1C is a snippet of $\sim 25 \mathrm{~ms}$, showing the propagation of motor activity captured along the electrodes in the array. Signals were captured with respect to a reference electrode located on the same muscle (ref in figure) and analysed in differential or bipolar mode. Using the voltage differences across the electrode locations along the column of the array $\left(D_{1}=E_{2 r}-E_{1 r} ; D_{2}=E_{3 r^{2}}-E_{2 r} ; D_{3}=E_{4 r^{-}}\right.$ $\mathrm{E}_{3 \mathrm{r}}$ ), we estimated the conduction velocity for the motor activity. Cross correlation analysis was done on a trial-by-trial basis across the session (see right panel in Fig 1C for example from a trial from a representative session) between $\mathrm{DF}_{1}$ and $\mathrm{DF}_{2}$ to measure the $\mathrm{lag}_{1}$, which was $0.8 \mathrm{~ms}$. Conduction velocity was estimated by dividing the average lag 1 for the session with the distance between the electrode pairs $\left(\mathrm{d}_{1}=2.5 \mathrm{~mm}\right)$. Similarly, conduction velocity for $\mathrm{d}_{2}=5 \mathrm{~mm}$ was estimated from the average lag2 which was measured from a cross correlation between $\mathrm{DF}_{1}$ and $\mathrm{DF}_{3}\left(\operatorname{lag}_{2}=2.3 \mathrm{~ms}\right.$, for the example trial from the representative session). The values for estimated conduction velocity were consistent across the population for different inter-electrode distances (hand: $\mathrm{d}_{1}=2.5 \mathrm{~mm}: \mathrm{cv}_{1}=1.24 \pm .28 \mathrm{~m} / \mathrm{s}$ and $\mathrm{d}_{2}=5.0 \mathrm{~mm}, \mathrm{cv}_{2}=$ $1.21 \pm .29 \mathrm{~m} / \mathrm{s}$ and cursor: $\mathrm{d}_{1}=2.5 \mathrm{~mm}: \mathrm{cv}_{1}=1.30 \pm .33 \mathrm{~m} / \mathrm{s}$ and $\mathrm{d}_{2}=5.0 \mathrm{~mm}, \mathrm{cv}_{2}=1.21 \pm .20$ $\mathrm{m} / \mathrm{s}$ ).

We analysed the EMG activity through two distinct methods: (a) the traditional rootmean square approach and (b) the raster-based approach. In the former approach, the envelope or variability in the EMG signal was captured using a running window over the 
405 signal squared (see methods, top panel: Fig 1E), whereas in the latter approach, EMG signals

406 were first decomposed into multi-unit activity, spike timings were convolved using a 407 temporal gaussian filter for obtaining a spike density function (see methods, bottom panel:

408 Fig 1E). Both approaches gave comparable results (Fig 1F; Hand: delayed task: Pearson's $r$ $409=0.91 \pm 0.04, \mathrm{p}<0.0001$ and immediate: Pearson's $\mathrm{r}=0.92 \pm 0.04, \mathrm{p}<0.0001$; Cursor:

410 delayed task: Pearson's $r=0.80 \pm 0.04, \mathrm{p}<0.0001$ and immediate task: Pearson's $r=0.86 \pm$ 411 0.01, $\mathrm{p}<0.0001)$. However, since the latter method provided information about the spike 412 waveforms in addition to the frequency of spike occurrence, we only used the latter analyses 413 henceforth, unless otherwise specified.

\section{Task-related early recruitment of motor unit activity}

The activity of the anterior deltoid (shoulder) muscle was recorded when the subjects reached for the target presented on the screen either by making actual hand movements or when they had to apply appropriate amount of force on the robotic arm to control the cursor (see methods). To assess whether motor activity was context dependent, the response of the muscle activity, for stimulus located in and out of the movement field was aligned on different events: go cue and movement onset for delayed versus immediate tasks for hand and cursor movements. An example of a motor activity (SDF) that shows differences in the recruitment pattern in the delayed versus immediate reach task is shown in Fig $2 \mathbf{A}$ (left vs. right panel). Early building up of activity (onset: $-591 \mathrm{~ms}$ ) or significant increase in recruitment of motor units (at go cue: $\Delta 7 . \pm 1 \mathrm{sp} / \mathrm{s}, \mathrm{t}(64)=6.49, \mathrm{p}<0.001$ ) prior to the go cue was seen in the delayed task condition, as shown for the example session. 7 out of 9 subjects showed similar trends during the delay period (onset: across population: $-153 \pm 60 \mathrm{~ms}$, $\mathrm{t}(8)=9.15, \mathrm{p}<.001)$ for the delayed reach task. Following the go cue and prior to movement onset further modulations were observed during the delayed reach task (Delayed task: onset following go cue: $227 \mathrm{~ms}$ for the example session; across population: $336 \pm 39 \mathrm{~ms}$ ). These subsequent modulations occurred earlier when compared to the immediate task (immediate task: onset: $394 \mathrm{~ms}$ for the example session; across population: $455 \pm 50 \mathrm{~ms}$; pairwise t-test: $\mathrm{t}(8)=4.82, \mathrm{p}=.001)$. Such context dependent early and late modulations in the motor activity for delayed versus immediate movements can be attributed to the presence or absence of a delay period.

In contrast, Fig 2B shows an example from one of the sessions where no significant 
437 cue: $\Delta 0.070 \pm 0.35 \mathrm{sp} / \mathrm{s}, \mathrm{t}(37)=0.20, \mathrm{p}=0.841)$. Only 1 out of 11 subjects showed a positive

438 difference in activity during the delay time from the baseline. Most of the subjects showed 439 recruitment of motor units after the go cue and just prior to movement onset. Similar to the 440 hand task; early onset in motor activity was seen following the go cue during reaction time 441 for the delayed task condition when compared to the immediate task condition (onsets: 442 delayed task: $211 \mathrm{~ms}$ for the example session; across population: $254.00 \pm 13.76 \mathrm{~ms}$ and 443 immediate task: $211 \mathrm{~ms}$ for the example session; across population: $301.54 \pm 23.66 \mathrm{~ms}$, 444 pairwise t-test: $\mathrm{t}(10)=1.75, \mathrm{p}=.05)$.

Fig 2C, summarises the results for modulations seen during the delay period relative to the go cue. Towards this purpose, the two metrics were used (i) activity level at the time of go cue and (ii) growth rate or slopes prior to go cue for delayed vs immediate condition for both hand and cursor tasks. The activity level at the go cue for hand movements, in the delayed task condition was higher when compared to the immediate task (delayed task: $22.28 \pm 3.89 \mathrm{sp} / \mathrm{s}$, immediate task: $18.05 \pm 3.51 \mathrm{sp} / \mathrm{s}$; paired t test: $\mathrm{t}(8)=-3.01, \mathrm{p}=0.016)$. However, no change in activity level at the time of go cue was seen across population for cursor movements (delayed task: $9.79 \pm 1.83 \mathrm{sp} / \mathrm{s}$, immediate task: $9.48 \pm 1.84 \mathrm{sp} / \mathrm{s}$; paired t test: $\mathrm{t}(10)=-0.264, \mathrm{p}=0.79)$. Next, to capture the ramping up of motor activity during the delay period, we measured growth rate or the slope of motor activity prior to the go cue. For hand movements, there was significant increase in growth rate captured across different sessions for the delay task relative to the immediate task condition (delayed task: $3.08 \pm 1.04 \mathrm{sp} / \mathrm{s}^{2}$, immediate task: $\left.0.20 \pm 1.04 \mathrm{sp} / \mathrm{s}^{2} ; \mathrm{t}(8)=-3.003 ; \mathrm{p}=0.017\right)$. However, no significant change in growth rate or ramping up of activity was seen for cursor movements (delayed task: $0.32 \pm 1.15 \mathrm{sp} / \mathrm{s}^{2}$, immediate task: $\left.-0.45 \pm 1.34 \mathrm{sp} / \mathrm{s}^{2 ;} \mathrm{t}(10)=-0.685 ; \mathrm{p}=0.508\right)$.

In addition to the pairwise differences between delayed and immediate task condition, differences in motor activity between hand and cursor movements were also observed as mentioned below. The population level activity at the time of go cue for cursor movements was significantly lower than the hand condition for both delayed (Hand: 22.28 $\pm 3.89 \mathrm{sp} / \mathrm{s}$; Cursor: $9.79 \pm 1.83 \mathrm{sp} / \mathrm{s}$, two sample t-test t-test: $\mathrm{t}(18)=3.08, \mathrm{p}=0.006)$ and immediate (Hand movements: $18.05 \pm 3.51 \mathrm{sp} / \mathrm{s}$; Cursor movements: $9.48 \pm 1.84 \mathrm{sp} / \mathrm{s}$, two sample t-test: $t(18)=2.27, p=0.035)$ tasks. Next, not surprisingly, when we compared the growth rate or slopes prior to the go cue for immediate task, in hand and cursor movement tasks, we found no significant differences between them (Hand: $0.20 \pm 1.04 \mathrm{sp} / \mathrm{s}^{2}$; Cursor: $-0.45 \pm 1.34 \mathrm{sp} / \mathrm{s}^{2}$, 
469

470

471

472

473

474

475

476

477

two sample t-test: $\mathrm{t}(18)=0.374, \mathrm{p}=0.71)$. However, for the delayed task condition, there was a significant difference in growth rate prior to the go cue between hand and cursor movements. The ramping up of activity during the delay period for hand movements was absent for cursor movements (Hand: $3.08 \pm 1.04 \mathrm{sp} / \mathrm{s}^{2}$; Cursor: $0.32 \pm 1.15 \mathrm{sp} / \mathrm{s}^{2}$, two sample t-test: $\mathrm{t}(18)=1.731$, $\mathrm{p}=0.05)$.

We next looked at differences in recruitment pattern, if any, between delayed versus immediate task conditions during the reaction time following the go cue (Fig 2D). Based on the motor activity prior to initiating movements, both metrics from the accumulator model (i) threshold or motor activity at movement onset and the (ii) growth rate following the go cue were used to look at differences in recruitment pattern during the delayed and immediate task conditions for hand and cursor-based movements. Motor activity when aligned on movement onset reached a particular threshold prior to initiation of movements. This threshold value was not significantly different for both the delayed and immediate task condition for hand and cursor movements (Threshold for hand movements: delayed task: $46.17 \pm 1.63 \mathrm{sp} / \mathrm{s}$, immediate task: $46.45 \pm 1.65 \mathrm{sp} / \mathrm{s}$, paired t test: $\mathrm{t}(8)=0.73, \mathrm{p}=0.48$; threshold for cursor movements: delayed task: $16.36 \pm 2.39 \mathrm{sp} / \mathrm{s}$, immediate task: $14.02 \pm 2.23 \mathrm{sp} / \mathrm{s}$; paired t test: $\mathrm{t}(10)=-1.97, \mathrm{p}=0.077)$. Also, the growth rate of motor activity for delayed versus immediate task conditions were not significantly different following the go cue and prior to movement initiation for both hand (growth rate: delayed task: $68.4 \pm 9.5 \mathrm{sp} / \mathrm{s}^{2}$, immediate task: $57.7 \pm 6.4$ $\mathrm{sp} / \mathrm{s}^{2}$; paired t test: $\mathrm{t}(8)=-2.04, \mathrm{p}=0.075$ ) and cursor based movements (growth rate: delayed task: $41.4 \pm 10.6 \mathrm{sp} / \mathrm{s}^{2}$, immediate task: $29.0 \pm 6.2 \mathrm{sp} / \mathrm{s}^{2}$; paired t test: $\left.\mathrm{t}(10)=-1.77, \mathrm{p}=0.106\right)$.

Furthermore, both the parameters related to initiation of movements remained similar for different task context following go cue. We noticed that across the population the threshold levels were significantly low for cursor movements in comparison to hand movements in both the delayed tasks (threshold: hand movements: $46.17 \pm 1.63 \mathrm{sp} / \mathrm{s}$; cursor movements: $16.36 \pm 2.39 \mathrm{sp} / \mathrm{s}$, two sample t-test: $\mathrm{t}(18)=9.80, \mathrm{p}<0.001)$ and immediate (threshold: hand movements: $46.45 \pm 1.65 \mathrm{sp} / \mathrm{s}$; cursor movements: $14.02 \pm 2.23 \mathrm{sp} / \mathrm{s}$, two sample t-test: $\mathrm{t}(18)=11.19, \mathrm{p}<0.001)$. Further, the growth rate or slope following the go cue was significantly steeper for hand movements when compared to cursor movements for delayed (growth rate: hand: $68.4 \pm 9.5 \mathrm{sp} / \mathrm{s}^{2}$; cursor: $41.4 \pm 10.6 \mathrm{sp} / \mathrm{s}^{2}$, two sample t-test: $\mathrm{t}(18)=1.849, \mathrm{p}=0.04$ ) and immediate movements (growth rate: hand: $57.7 \pm 6.4 \mathrm{sp} / \mathrm{s}^{2}$; cursor: $29.0 \pm 6.2 \mathrm{sp} / \mathrm{s}^{2}$, two sample t-test: $\left.\mathrm{t}(18)=3.19, \mathrm{p}=0.005\right)$. 
Spatial and temporal information encoded during the early recruitment of motor unit activity

We first asked whether the motor activity during the delay period contained any spatial information about the upcoming movement. We first characterized the movement field (MF) of the muscle activity as the direction with maximum activity, -125 to $25 \mathrm{~ms}$ relative to movement onset (Fig S1 and Fig S3, link: https://doi.org/10.5281/zenodo.6026951). The average directional tuning across the population for the anterior deltoid muscle (Hand: $109^{0}$ and Cursor: $103^{\circ}$ and $104^{\circ}$ ), indicated a right-upward component, as expected from a shoulder flexor (Fig S4). To calculate the time when motor activity could predict the direction of an upcoming movement, we first divided the trials into 2 groups: trials where movement went into the MF (in-MF) and trials where the movements were made outside the MF (out-MF). An ROC analysis for hand and cursor movements between in-MF and out-MF responses, for each time bin during visual, delay and movement epochs was performed to calculate the area under the curve statistic (AUC). AUC values range between 0.5 (no separability) to 1 (complete separability). Fig 3A shows a representative session, wherein motor activity showed modulation for in-MF response compared to the out-MF direction for the delayed and immediate task (left vs right). As shown in the panel below, the AUC value showed an increasing trend prior to the go cue (increase at go cue: $\Delta 0.142$ from baseline; onset: $-538 \mathrm{~ms}$ ) during the delay period in the delayed task. In the case of cursor movements (see Fig 3B), the responses of the muscle for in-MF and out-MF conditions during the delay epoch, were comparable and showed no such trend or modulation (at go cue: $\Delta 0.02$ from baseline): AUC or separability between in-MF and out MF responses for cursor movements was almost 0.5 .

Fig 3C summarizes the results to capture the trend across the population for hand movements. The discriminability between in-MF and out-MF responses increased continuously with time during the delay period between target onset to go cue for delayed hand movements only (see inset, $\triangle \mathrm{AUC}=0.05 \pm 0.02 ; \mathrm{t}(8)=2.33 ; \mathrm{p}=0.04$ ). Also, AUC exhibited a monotonically increasing trend which began at $-289 \pm 46 \mathrm{~ms}$ before the go cue (see methods). Even though the AUC values during the delay times were low $(<0.7)$,

530 discrimination times at these levels were observed for each subject. We also observed that following the presentation of the go cue, the AUC levels increased further towards unity 
533 The beginning of the post go cue discriminability appeared to reflect a second phase, which

534 was estimated by using a higher threshold value of 0.7 . Interestingly, the onset for the 535 average direction discrimination time for the delayed task occurred earlier when compared to 536 immediate hand movement task for this post go cue epoch as well (delayed task: $250.89 \pm 48$ 537 ms, immediate task: $363 \pm 48$ ms; pairwise $\mathrm{t}$ test: $\mathrm{t}(8)=7.05, \mathrm{p}<0.001)$.

Fig 3D summarizes the results across the population for cursor movements. The AUC value remained largely unchanged for the delayed cursor movement task until the go cue appeared (cursor: $\triangle \mathrm{AUC}=0.002 \pm 0.01 ; \mathrm{t}(10)=0.19 ; \mathrm{p}=0.851$ ). Following the go cue, the direction discriminability increased monotonically during the reaction time. For the same threshold level (AUC=0.7), an earlier onset in direction discrimination time was detected for the delayed task when compared to the immediate task (delayed task: $317 \pm 42 \mathrm{~ms}$; immediate task: $436 \pm 39 \mathrm{~ms}$; pairwise t-test: $\mathrm{t}(10)=4.37, \mathrm{p}<0.001)$. Taken together, these analyses indicate spatial information about upcoming movements were available to the peripheral musculature during the delay period for hand movements but were absent for cursor movements.

To test whether motor activity could explain the variability in reaction time (RT), we calculated a Pearson's correlation coefficient on trial-by-trial basis between motor unit activity and reaction time, both before and after the go cue, for delayed and immediate hand and cursor movements. Fig 4A and Fig 4B, shows response profile of motor activity for different trial groups binned based on fast to slow reaction times for a representative session

553 from hand and cursor movements, respectively. As hand movements showed modulation during the delay period, a window of $125 \mathrm{~ms}$ prior to go cue and $20 \mathrm{~ms}$ after the go cue, was considered for the analysis to check if motor activity contained any temporal information about the upcoming movements. Consistent with this notion, we observed that the ramp up of activity during the delay time was negatively correlated $\left(\mathrm{r}_{\mathrm{Go}}=-0.382 ; \mathrm{p}=0.001\right.$, see inset in Fig 4A) with reaction time. However, no correlation was seen during the delay time for cursor movements $\left(\mathrm{r}_{\mathrm{Go}}=0.10 ; \mathrm{p}=0.55\right.$; see inset in Fig 4B). Furthermore, the negative correlations became stronger and significant at single trial levels for both delayed and

561 immediate hand movements between $175 \mathrm{~ms}$ to $325 \mathrm{~ms}$ following the go cue (Fig 4C; 562 delayed: $r_{R T}=-0.394 ; p=0.001$; immediate: $\left.r_{R T}=-0.528, p<0.001\right)$. Shown in Fig 4D is a 563 similar correlation plot for the representative session but for cursor movements (delayed: $r_{R T}$ $564=-0.546 ; \mathrm{p}<0.001 ;$ immediate: $\left.\mathrm{r}_{\mathrm{RT}}=-0.071, \mathrm{p}=0.66\right)$. 
565 Fig 4E summarises the results seen above across the population for hand movements.

566 Change in activity from baseline prior to and at the time of go cue was significant during the 567 delay task when compare to immediate task condition (delayed: $\mathrm{r}=-0.19 \pm .04 ; \mathrm{t}(8)=-4.82 ; \mathrm{p}$ $568<0.01$; immediate: $\mathfrak{t}(8)=0.166 ; \mathrm{p}=0.87$ ). One of the interesting things we noted was that 569 following the go cue, the correlations became stronger, but was less negative for the delayed 570 hand movement task (hand: $\mathrm{r}=-0.44 \pm .06 ; \mathrm{t}(8)=-6.75 ; \mathrm{p}<0.001$ ) compared to the immediate 571 task (hand: $\mathrm{r}=-0.36 \pm .05 ; \mathrm{t}(8)=-7.93 ; \mathrm{p}<0.001$ ). Like Fig 4E, Fig 4F summarises the results 572 across the population for cursor movements. As no modulation was seen during the delay 573 time for cursor movements, the activity prior to go cue was not correlated with reaction times 574 (delayed: $\mathrm{t}(10)=-0.74 ; \mathrm{p}=0.47$; immediate: $\mathrm{t}(10)=-0.29 ; \mathrm{p}=0.77$ ). However, as expected, 575 prior to movement onset, the correlations with reaction time grew stronger for both delayed 576 and immediate cursor tasks (delayed task: $\mathrm{r}=-0.28 \pm .08 ; \mathrm{t}(10)=-4.60 ; \mathrm{p}<0.001$; immediate 577 task: $\mathrm{r}=-0.26 \pm .06 ; \mathrm{t}(10)=-3.53 ; \mathrm{p}<0.001)$.

To summarise, during the delay period, both spatial and temporal information reached periphery prior to the go cue for hand movements only. For cursor movements since no significant modulation was seen during the delay period, behaviouraly relevant spatial and temporal information was not present. However, and not surprisingly, the build-up in muscle activity could be used to discriminate the upcoming direction of movement, after the go cue but prior to movement onset.

Motor unit activity can be described by accumulate to threshold models of response

\section{initiation}

Next, we tested whether accumulator models could be used to assess initiation of movements from shoulder muscles during the reaction time in hand and cursor task. In previous studies (Hanes and Schall 1996) such ramping activity during reaction time has been formalized in accumulator models which has been used only in the context of immediate movements to understand the neural basis of response preparation. Here, we tested if these parameters describing accumulation could be extended to understand initiation of hand and cursor movements. The response for each subject was divided into slow and fast reaction times (see methods). Different parameters of the accumulator model- (i) activity at the time of go cue (ii) slopes and (iii) onset for activity during the delay period (iv) and threshold or 
596 motor activity at the time of movement initiation were calculated for each session under both the conditions (see method).

The response from a representative session for a delayed and immediate hand movement task is shown in Fig $\mathbf{5 A}$ and Fig 5B, respectively, for slow and fast reaction times (see methods) aligned on go cue and reaction time. For the delayed but not the immediate task, the activity around the go cue was higher for fast reaction times compared to slow reaction times. This observation was consistent across the population (delayed: fast reaction time: $47.54 \pm 7.82 \mathrm{sp} / \mathrm{s}$, slow reaction time: $41.43 \pm 7.88 \mathrm{sp} / \mathrm{s} ; \mathrm{t}(8)=-3.00, \mathrm{p}<0.01$; first panel in Fig 5C; immediate: fast reaction time: $35.58 \pm 7.04 \mathrm{sp} / \mathrm{s}$, slow reaction time: $36.41 \pm 7.26$ $\mathrm{sp} / \mathrm{s} ; \mathrm{t}(8)=0.677, \mathrm{p}=0.25$; first panel in Fig 5D). We found no significant changes in motor activity onset times between fast and slow reaction times for the delayed (fast RT $150 \pm 43$ ms; slow RT $145 \pm 37$ ms; (t $(8)=0.18, p=0.43$; second panel in Fig 5C) and immediate task (fast RT $212 \pm 34 \mathrm{~ms}$ and slow RT $246.66 \pm 28.00 \mathrm{~ms} ;(\mathrm{t}(8)=0.49, \mathrm{p}=0.31$; second panel in Fig 5D). However, the change in activity at the time of the go cue was related to systematic changes in the rate at which the activity rose during reaction time. Across the population, we observed significant changes in growth rate for both delayed (fast reaction time: $220.38 \pm$ $37.00 \mathrm{sp} / \mathrm{s}$, slow reaction time: $170.78 \pm 21.42 \mathrm{sp} / \mathrm{s} ; \mathrm{t}(8)=-2.56, \mathrm{p}=0.03$; third panel in Fig 5C) as well as immediate movements (fast reaction time: $223.08 \pm 23.74 \mathrm{sp} / \mathrm{s}$, slow reaction time: $158.94 \pm 21.34 \mathrm{sp} / \mathrm{s}, \mathrm{t}(8)=-3.21, \mathrm{p}=0.006$; third panel in Fig 5D). In addition, we also aligned the response on movement onset and tested whether threshold activity at the time of movement onset changed for fast and slow reaction time during delayed and immediate task conditions. Threshold activity in both the tasks, under delayed and immediate conditions was similar for slow and fast reaction times. This observation was consistent across the population (delayed: fast reaction time: $94.26 \pm 3.14 \mathrm{sp} / \mathrm{s}$, slow reaction time: $89.84 \pm 3.99 \mathrm{sp} / \mathrm{s} ; \mathrm{t}(8)=$ .004, p=0.49; fourth panel in Fig 5C; and immediate: fast reaction time: $92.71 \pm 3.53 \mathrm{sp} / \mathrm{s}$, slow reaction time: $93.25 \pm 3.23 \mathrm{sp} / \mathrm{s} ; \mathrm{t}(8)=.004, \mathrm{p}=0.49$; fourth panel in Fig 5D).

We also tested whether the same parameters could be used to explain the motor activity pattern for cursor movements during delayed and immediate conditions. Interestingly, unlike the growth rate, which was a dominant factor for the hand movement task, onsets were the dominating factor in cursor movements. Fig 6A and Fig $6 \mathbf{B}$ shows the response from a representative cursor session for a delayed and immediate task, for slow and fast reaction 
628 slow and fast reaction times did not change and this observation was consistent across the population (delayed: fast reaction time: $36.79 \pm 7.58 \mathrm{sp} / \mathrm{s}$, slow reaction time: $37.10 \pm 6.56$ $\mathrm{sp} / \mathrm{s} ; \mathrm{t}(10)=0.14, \mathrm{p}=0.44$; first panel in Fig 6C; immediate: fast reaction time: $35.69 \pm 7.25$ $\mathrm{sp} / \mathrm{s}$, slow reaction time: $37.15 \pm 6.99 \mathrm{sp} / \mathrm{s} ; \mathrm{t}(10)=0.95, \mathrm{p}=0.18$; first panel in Fig 6D). Interestingly, we found significant increase in onset times for fast and slow reaction times for delayed and immediate task conditions (delayed task condition (fast reaction time: $196.00 \pm$ $22.47 \mathrm{~ms}$ and slow reaction time: $323.27 \pm 33.07 \mathrm{~ms}$, t-test: $\mathrm{t}(10)=4.19, \mathrm{p}<0.001$, second panel in Fig 6C; immediate task condition: fast reaction time: $244.36 \pm 34.10 \mathrm{~ms}$ and slow reaction time: $425.90 \pm 36.92 \mathrm{~ms}, \mathrm{t}(10)=5.37, \mathrm{p}<0.001$; second panel in Fig 6D). However, we did not find any significant changes in growth rates for the delayed and immediate conditions (Delayed task condition: fast reaction time: $182.42 \pm 30.58 \mathrm{sp} / \mathrm{s}$ and slow reaction time: $149.18 \pm 23.14 \mathrm{sp} / \mathrm{s}$; t-test: $\mathrm{t}(10)=-1.66, \mathrm{p}=0.41$, third panel in Fig 6C; Immediate task condition: fast reaction time: $88.87 \pm 24.36 \mathrm{sp} / \mathrm{s}$ and slow reaction time: $140.46 \pm 26.70 \mathrm{sp} / \mathrm{s}$; t-test: $t(10)=1.69, \mathrm{p}=0.063$; third panel in Fig 6D). In addition, we also aligned population response on movement onset and tested whether threshold activity at the time of movement onset changed for fast and slow reaction time during delayed and immediate task condition. Activity in both the tasks, under delayed and immediate conditions was similar for slow and fast reaction times. This observation was consistent across the population (delayed: fast reaction time: $51.09 \pm 8.63 \mathrm{sp} / \mathrm{s}$, slow reaction time: $54.60 \pm 7.63 \mathrm{sp} / \mathrm{s} ; \mathrm{t}(10)=1.66, \mathrm{p}=0.063$; fourth panel in Fig 6C; immediate: fast reaction time: $47.33 \pm 8.71 \mathrm{sp} / \mathrm{s}$, slow reaction time: $51.05 \pm 7.76 \mathrm{sp} / \mathrm{s} ; \mathrm{t}(10)=1.66, \mathrm{p}=0.0 .63$; fourth panel in Fig 6D).

In summary, by analysing the data for different task context- delayed and immediate task, we tested the applicability of the linear accumulator model and found that different parameters/architectures explained the modulations of motor activity during the reaction time for both hand and cursor studies (Fig 5, Fig 6). Both, growth rate and onsets played an important role during the reaction time to help initiate an upcoming movement. 
655

656

657

658

659

660

661

662

\section{Discussion}

Our study is the first attempt at using high density surface EMG to show the presence of early recruitment of muscle activity that is correlated with reaction time and is also task dependent. Our results show both spatial and temporal information is encoded in motor activity during the delay period for hand movements but not for isometric cursor movements. However, after the go cue, irrespective of the type of movement (hand or cursor), the activity during the reaction time could be used to predict the variability in time to movement onset with respect to delayed and immediate tasks.

\section{Context dependence of early recruitment of motor activity}

A major unresolved issue pertaining to the skeletal motor system is whether motor preparation is reflected in the peripheral nervous system during an instructed delay time task as has been observed centrally. Seemingly different results for the same task performed but under slightly different conditions have been observed by different groups. Prut and Fetz (1999) recorded from distal muscle groups while macaques were involved in an isometric force task and they showed no modulation of EMG activity in muscles during the delay period despite being present in spinal cord neurons. However, while macaques were involved in making actual flexion-extension arm movements, Mellah et al. 1990 observed motor units are recruited during the delay period in proximal muscle groups. More recently, in humans Papaioannou and Dimitriou 2021, also observed preparatory signals in Ia afferent but in the absence of changes in EMG activity. These disparate results could be a consequence of the different muscles used since proximal versus distal muscle groups have different delays in muscle activation which contribute partly to the observed transport delay between reach and grasp actions observed in overt behaviour (Jeannerod 1981). Thus, differences in proximal versus distal muscles may be a consequence of these muscle groups being subject to different levels of spinal inhibitory control.

However, our results show that delay period activity is also dependent on task context for the same muscle. We observed no activity into the periphery despite the muscle being subject to forces that were comparable to that generated for natural reaching movements (see Fig S2). We interpret these results as suggesting that the absence of early recruitment in isometric movements might be a consequence of the absence of explicit kinematic planning during natural movements. These results also align with Papaioannou and Dimitriou 2021; Prut and Fetz 1999, since their task was cursor based and may not have necessitated explicit 
687 kinematic planning. In contrast, dynamics related activity of motor units that typically would 688 dominate EMG activity, is likely to be subject to stronger inhibitory control and this could be 689 one of the reasons for not seeing information leaking through into the peripheral nervous 690 system during isometric movements that are associated with a dynamic component. In this 691 respect our results are consistent with both Mellah et al. 1990; Prut and Fetz 1999.

\section{Early recruitment of muscle activity encodes spatial and temporal information}

Previous studies in psychology have suggested that information can flow in a continuous mode from successive processing stages (Mcclelland 1979; Meyer et al. 1988). Similarly, studies in the oculomotor and motor systems have suggested that information can flow in a continuous mode from different brain areas that are involved in visual to movement transformations (Bichot et al. 2001; Cisek and Kalaska 2005; Crammond and Kalaska 2000; Schmolesky et al. 1998). We extended these ideas to study flow of information into the peripheral musculature. Previous efforts have tried to address this issue in the case of immediate movements and have shown evidence of a stimulus linked response (SLR) in proximal arm and neck muscles (Corneil and Munoz 2014; Pruszynski et al. 2010) that may be associated with neural activity associated with the reticulospinal tract (Kearsley et al. 2021). However, since the SLR is mostly associated with reflexive movements, dissociating early and late components of movement planning from execution are challenging. By separating processes pertaining to where and when an action is to be made by using a delay period in the visually guided delay time paradigm, we now show that slower modulations in the EMG signal carried both spatial and temporal information about a prospective movement much before the go cue, independent of the occurrence of an SLR.

The presence of both early and late components of motor planning could be seen in the AUC and temporal correlations (Fig 3 and 4). Whereas during the delay period, the AUC and temporal correlations with reaction times were modest, they dramatically increased after the go period. This is not surprising, given the presence of variability after the go cue is more is expected to dilute the contributions of preparatory activity to reaction times. Nevertheless, what was surprising is the absence of such early modulations for cursor movements only.

715 Such specificity with respect to task context suggests that different aspects of motor planning 716 are reflected in the proximal muscle groups. 
Although the specific role of such early muscle activity is unclear, such early muscle activity may allow the shoulder muscles to overcome the larger inertia of the limb to facilitate the initiation of hand movements. Such early activity could therefore either prime the proximal muscle groups, or drive or even control the subsequent dynamics related muscle activation as envisioned in equilibrium control models (Dimitriou and Edin 2010; Latash 2018; Polit and Bizzi 1979). Further, if such early activity is related to kinematics, it is expected to be spatially specific, as we observed for the agonist muscle, but not when the same muscle was an antagonist. The rather early activity observed in the absence of overt movement further suggests that this activity is likely to reflect the activation of small motor units, which may explain why their contribution in whole muscle EMG activity may have gone unnoticed. Although speculative, based on the bilateral activation of the stimulus linked responses recently observed by (Kearsley et al. 2021), we propose that such early activity may reflect the input from the reticular spinal tract that could be the source of gamma unit activation that is being assessed by the high-density surface EMG electrodes used in this study. Alternatively such early kinematic activity may also reflect contributions of the parietal cortex that have been recently shown to project to neurons in ventral spinal cord (Rathelot et al. 2017).

Assessing initiation of movements with an accumulator model that tracks motor activity recruitment

A Linear Accumulation to Threshold with Ergodic Rate (LATER) model has been used to successfully account for reaction time data in eye and hand movement tasks (Asrress and Carpenter 2001; Carpenter and Williams 1995; Dean et al. 2011; Gopal and Murthy Jana et al. 2017). The use of a LATER model was largely motivated by its relative simplicity and the ability to quantify the parameters that describe motor recruitment and its association with movement initiation and not necessarily indicative of the underlying neural mechanisms per se. Indeed, recording of the neurons in the primary and premotor cortex, unlike those in the oculomotor system (Dorris et al. 1997; McPeek and Keller 2002a, 2002b; Murthy et al. 2007;

744 Nelson et al. 2016; Thompson et al. 1997), are better understood as emerging from a 745 population of activity distributed across brain areas as part of a dynamic ensemble 746 (Churchland et al. 2010; Georgopoulos et al. 1986; Kaufman et al. 2014). Here we used the 747 LATER model to test whether the model can provide a framework that links movement 748 initiation to the recruitment of motor units in the periphery. As expected from the early delay 
749 period activity, the correlations between the parameters of the model and reaction times were

750 modest but consistent and significant within and across subjects. However, and not

751 surprisingly, the correlations became much stronger, the close the activity was to movement

752 initiation. To our knowledge, this approach has been used for the first time, to study how

753 accumulation to threshold models (LATER) can be used to assess whether the recruitment of

754 motor activity observed in the periphery is linked in a systematic manner to behaviour.

By recording from motor activity using high density surface arrays in the shoulder muscle, we also tested the generality of our findings across tasks. Longer reaction times in both tasks (delayed and immediate task), in the hand and cursor studies was associated by a 758 decrease in slope. However, we also noticed some difference between the recruitment 759 patterns associated with delayed and immediate movements. The delay period in the delay task for hand movement showed modulations which could be used to prepare for the upcoming movements and hence it was not surprising that faster reaction times was associated with a increase in the baseline activity at the time of the GO cue. However, we also observed some differences between the two tasks, with the slope being the significant parameter for explaining RT differences for hand movements but not for cursor movements. Interestingly, onset was the consistent factor that could explain ensuing RT changes for delayed and immediate tasks during cursor movements, despite the same activity level at the time of GO cue. This implies that the parameters of the LATER model were able to capture the nuances in the pattern of putative motor unit activity that contributed towards changes in RT. Where slope was the important parameter for hand studies that could explain variability in RT, onsets played a consistent part in both the task contexts (delayed and immediate movements) for the cursor studies. Further, we also found that threshold activity at the time of movement initiation remained the same for delayed and immediate movements across RT as per the predictions of the LATER model, but this value differed depended on whether the condition was a cursor or hand movement. This in effect is interesting as task specific affects

775 have also been reported in different brain areas for eye and hand movements (Basu et al.

776 2021; Mushiake et al. 2009; Schlag-Rey et al. 1997; Tanji et al. 2007).

\section{7}

\section{Methodological Issues}

It is noteworthy and rather striking that the results we obtained have not been documented in prior EMG studies in humans of reaching movements using either surface EMG or even single unit recordings of motor units. We believe several factors may have 
781 contributed to our ability to detect these subtle changes that may have gone unnoticed in prior

782 work. First, most of the previous studies have used global EMG measures such as root mean

783 square (rms). These methods are generally crude and not sensitive enough to detect local or 784 small changes in the muscle response (as seen in log scale, see inset Fig 1E), and thus, 785 evidence of early recruitment may have been missed. Our results suggest that the noise or the 786 variability present in the RMS has certain features which could be exploited by a thresholding 787 operation to isolate point processes that more closely align with the motor unit recruitment. 788 However, an obvious shortfall of such a thresholding approach would be to render the data 789 not sensitive to decreases in motor activity. Perhaps, such a bias would have the fortuitous 790 effect of amplifying any underlying recruitment that would be otherwise obscured by decrements in EMG activity. Second, the use of spatial filtering techniques for high-density surface array EMG recordings is known to selectively enhance local voltage changes which would be more likely to reflect the recruitment patterns of motor units (Gazzoni et al. 2004; Luca et al. 2006; Mambrito and De Luca 1984; Masuda and De Luca 1991; Merletti and Farina 2009; Rau and Disselhorst-Klug 1997) rather than global muscle activity; and our use of template matching and the measurement of the propagation of the signal along the muscle fibres suggests this to be the case. Third, unlike many previous reports, our study focussed on proximal shoulder muscles that may be preferentially targeted by the reticulospinal system that may be responsible for the observed delayed activity since these muscles also robustly express a stimulus locked response (Kearsley et al. 2021). Finally, since this early activity was correlated with reaction time, had spatially selective response fields (see Fig S4) and was only observed for delayed movements but not for cursor movements generated by isometric muscle force, it cannot be an artifact.

\section{Acknowledgement:}

This study was supported by an Intensification of Research in High Priority Areas Grant from the Department of Science and Technology, Government of India; a Department of Biotechnology - Indian Institute of Science (DBT-IISc) phase 2 partnership programme grant; and institutional support from the Ministry of Human Resource Development to A.M. and S.P.R. were supported by scholarship by Indian Institute of Science, Bangalore. 
bioRxiv preprint doi: https://doi.org/10.1101/2022.02.09.479690; this version posted February 14,2022 . The copyright holder for this preprint (which was not certified by peer review) is the author/funder, who has granted bioRxiv a license to display the preprint in perpetuity. It is made available under aCC-BY-NC-ND 4.0 International license.

\section{Author Contributions}

813 S.P.R. and A.M. designed the experiments, S.P.R. performed the experiments and analysed

814 the data and both the authors contributed to writing the manuscript. 


\section{References}

817 Alexander GE, Crutcher MD, DeLong MR. Chapter 6 Basal ganglia-thalamocortical circuits: Parallel 818 substrates for motor, oculomotor, "prefrontal" and "limbic" functions. Prog Brain Res 85: 119-146, 8191991.

820 Asrress KN, Carpenter RH. Saccadic countermanding: a comparison of central and peripheral stop 821 signals. Vision Res 41: 2645-2651, 2001.

822 Basu D, Sendhilnathan N, Murthy A. Neural mechanisms underlying the temporal control of 823 sequential saccade planning in the frontal eye field. Proc Natl Acad Sci U S A 118: e2108922118, 8242021.

825 Bichot NP, Rao SC, Schall JD. Continuous processing in macaque frontal cortex during visual search. 826 Neuropsychologia 39: 972-982, 2001.

827 Bonnet M, Requin J, Semjen A. Human reflexology and motor preparation. Exerc Sport Sci Rev 9: 828 119-157, 1981.

829 Brunia $\mathrm{CH}$. Motor preparation: changes in amplitude of Achilles tendon reflexes during a fixed 830 foreperiod of one second. Psychophysiology 20: 658-664, 1983.

831 Burle B, Vidal F, Tandonnet C, Hasbroucq T. Physiological evidence for response inhibition in choice 832 reaction time tasks. Brain Cogn 56: 153-164, 2004.

833 Carpenter RH, Williams ML. Neural computation of log likelihood in control of saccadic eye 834 movements. Nature 377: 59-62, 1995.

835 Churchland MM, Cunningham JP, Kaufman MT, Ryu SI, Shenoy KV. Cortical Preparatory Activity: 836 Representation of Movement or First Cog in a Dynamical Machine? Neuron 68: 387-400, 2010.

837 Cisek $\mathbf{P}$, Kalaska JF. Neural correlates of reaching decisions in dorsal premotor cortex: specification 838 of multiple direction choices and final selection of action. Neuron 45: 801-814, 2005.

839 Cohen O, Sherman E, Zinger N, Perlmutter S, Prut Y. Getting ready to move: transmitted 840 information in the corticospinal pathway during preparation for movement. Curr Opin Neurobiol 20: $841696-703,2010$.

842 Corneil BD, Munoz DP. Overt Responses during Covert Orienting. Neuron 82: 1230-1243, 2014.

843 Crammond DJ, Kalaska JF. Prior information in motor and premotor cortex: activity during the delay 844 period and effect on pre-movement activity. J Neurophysio/ 84: 986-1005, 2000.

845 Davranche K, Tandonnet C, Burle B, Meynier C, Vidal F, Hasbroucq T. The dual nature of time 846 preparation: neural activation and suppression revealed by transcranial magnetic stimulation of the 847 motor cortex. Eur J Neurosci 25: 3766-3774, 2007.

848 Dean HL, Martí D, Tsui E, Rinzel J, Pesaran B. Reaction time correlations during eye-hand 849 coordination: behavior and modeling. J Neurosci Off J Soc Neurosci 31: 2399-2412, 2011.

850 Dimitriou M, Edin BB. Human muscle spindles act as forward sensory models. Curr Biol CB 20: 1763851 1767, 2010. 
852 Dorris MC, Munoz DP. Saccadic probability influences motor preparation signals and time to

853 saccadic initiation. J Neurosci Off J Soc Neurosci 18: 7015-7026, 1998.

854 Dorris MC, Paré M, Munoz DP. Neuronal activity in monkey superior colliculus related to the 855 initiation of saccadic eye movements. J Neurosci Off J Soc Neurosci 17: 8566-8579, 1997.

856 Duclos Y, Schmied A, Burle B, Burnet H, Rossi-Durand C. Anticipatory changes in human 857 motoneuron discharge patterns during motor preparation. J Physiol 586: 1017-1028, 2008.

858 Fautrelle L, Prablanc C, Berret B, Ballay Y, Bonnetblanc F. Pointing to double-step visual stimuli from 859 a standing position: very short latency (express) corrections are observed in upper and lower limbs 860 and may not require cortical involvement. Neuroscience 169: 697-705, 2010.

861 Gazzoni M, Farina D, Merletti R. A new method for the extraction and classification of single motor 862 unit action potentials from surface EMG signals. J Neurosci Methods 136: 165-177, 2004.

863 Georgopoulos AP, Schwartz AB, Kettner RE. Neuronal population coding of movement direction. 864 Science 233: 1416-1419, 1986.

865 Gopal A, Murthy A. Eye-hand coordination during a double-step task: evidence for a common 866 stochastic accumulator. J Neurophysiol 114: 1438-1454, 2015.

867 Graybiel AM. Neurotransmitters and neuromodulators in the basal ganglia. Trends Neurosci 13: $868 \quad 244-254,1990$.

869 Hanes DP, Patterson WF, Schall JD. Role of frontal eye fields in countermanding saccades: visual, 870 movement, and fixation activity. J Neurophysiol 79: 817-834, 1998.

871 Hanes DP, Schall JD. Neural control of voluntary movement initiation. Science 274: 427-430, 1996.

872 Hasbroucq T, Osman A, Possamaï CA, Burle B, Carron S, Dépy D, Latour S, Mouret I. Cortico-spinal

873 inhibition reflects time but not event preparation: neural mechanisms of preparation dissociated by 874 transcranial magnetic stimulation. Acta Psychol (Amst) 101: 243-266, 1999.

875 Hikosaka O, Wurtz RH. Visual and oculomotor functions of monkey substantia nigra pars reticulata.

876 II. Visual responses related to fixation of gaze. J Neurophysiol 49: 1254-1267, 1983a.

877 Hikosaka $\mathbf{O}$, Wurtz RH. Visual and oculomotor functions of monkey substantia nigra pars reticulata.

878 IV. Relation of substantia nigra to superior colliculus. J Neurophysiol 49: 1285-1301, 1983b.

879 Jana S, Gopal A, Murthy A. A Computational Framework for Understanding Eye-Hand Coordination. 880 J Indian Inst Sci 97: 543-554, 2017.

881 Jeannerod. Intersegmental coordination during reaching at natural visual objects. Atten Perform IX $882153-168,1981$.

883 Kaufman MT, Churchland MM, Ryu SI, Shenoy KV. Cortical activity in the null space: permitting 884 preparation without movement. Nat Neurosci 17: 440-448, 2014.

885 Kearsley SL, Cecala AL, Kozak RA, Corneil BD. Express Arm Responses Appear Bilaterally on Upper886 limb Muscles in an Arm Choice Reaching Task. bioRxiv: 2021.09.24.461726, 2021. 
887 Kettner RE, Schwartz AB, Georgopoulos AP. Primate motor cortex and free arm movements to

888 visual targets in three- dimensional space. III. Positional gradients and population coding of

889 movement direction from various movement origins. J Neurosci 8: 2938-2947, 1988.

890 Latash ML. Muscle coactivation: definitions, mechanisms, and functions. J Neurophysiol 120: 88-

891 104, 2018.

892 Leach JC, Carpenter RH. Saccadic choice with asynchronous targets: evidence for independent

893 randomisation. Vision Res 41: 3437-3445, 2001.

894 Lecas JC, Requin J, Anger C, Vitton N. Changes in neuronal activity of the monkey precentral cortex

895 during preparation for movement. J Neurophysiol 56: 1680-1702, 1986.

896 Logan GD. On the ability to inhibit thought and action: a theory of an act of control. Psychol Rev 91:

$897 \quad 295-327,1984$.

898 Luca CJD, Adam A, Wotiz R, Gilmore LD, Nawab SH. Decomposition of Surface EMG Signals. J

899 Neurophysiol 96: 1646-1657, 2006.

900 Mambrito B, De Luca CJ. A technique for the detection, decomposition and analysis of the EMG

901 signal. Electroencephalogr Clin Neurophysiol 58: 175-188, 1984.

902 Masuda T, De Luca CJ. Technique for detecting MUAP propagation from high-threshold motor units.

903 J Electromyogr Kinesiol Off J Int Soc Electrophysiol Kinesiol 1: 75-80, 1991.

904 Mcclelland JL. On the time relations of mental processes: An examination of systems of processes in 905 cascade. Psychol Rev , 1979.

906 McGill KC, Lateva ZC, Marateb HR. EMGLAB: an interactive EMG decomposition program. J Neurosci 907 Methods 149: 121-133, 2005.

908 McPeek RM, Keller EL. Saccade target selection in the superior colliculus during a visual search task. 909 J Neurophysiol 88: 2019-2034, 2002a.

910 McPeek RM, Keller EL. Superior colliculus activity related to concurrent processing of saccade goals 911 in a visual search task. J Neurophysio/ 87: 1805-1815, 2002b.

912 Mellah S, Rispal-Padel L, Riviere G. Changes in excitability of motor units during preparation for 913 movement. Exp Brain Res 82: 178-186, 1990.

914 Merletti R, Farina D. Analysis of intramuscular electromyogram signals. Philos Trans $R$ Soc Math Phys 915 Eng Sci 367: 357-368, 2009.

916 Meyer DE, Osman AM, Irwin DE, Yantis S. Modern mental chronometry. Biol Psychol 26: 3-67, 1988.

917 Mink JW. THE BASAL GANGLIA: FOCUSED SELECTION AND INHIBITION OF COMPETING MOTOR 918 PROGRAMS. Prog Neurobiol 50: 381-425, 1996.

919 Moran DW, Schwartz AB. Motor cortical representation of speed and direction during reaching. J

920 Neurophysiol 82: 2676-2692, 1999.

921 Murthy A, Ray S, Shorter SM, Priddy EG, Schall JD, Thompson KG. Frontal eye field contributions to 922 rapid corrective saccades. J Neurophysiol 97: 1457-1469, 2007. 
923 Mushiake H, Sakamoto K, Saito N, Inui T, Aihara K, Tanji J. Involvement of the prefrontal cortex in 924 problem solving. Int Rev Neurobiol 85: 1-11, 2009.

925 Nelson MJ, Murthy A, Schall JD. Neural control of visual search by frontal eye field: chronometry of 926 neural events and race model processes. J Neurophysiol 115: 1954-1969, 2016.

927 Noorani I, Carpenter RHS. The LATER model of reaction time and decision. Neurosci Biobehav Rev 928 64: 229-251, 2016.

929 Papaioannou S, Dimitriou M. Goal-dependent tuning of muscle spindle receptors during movement 930 preparation. Sci Adv 7: eabe0401, 2021.

931 Parent A, Hazrati L-N. Functional anatomy of the basal ganglia. I. The cortico-basal ganglia-thalamo932 cortical loop. Brain Res Rev 20: 91-127, 1995.

933 Perfiliev S, Isa T, Johnels B, Steg G, Wessberg J. Reflexive limb selection and control of reach

934 direction to moving targets in cats, monkeys, and humans. J Neurophysiol 104: 2423-2432, 2010.

935 Polit A, Bizzi E. Characteristics of motor programs underlying arm movements in monkeys. J

936 Neurophysiol 42: 183-194, 1979.

937 Prochazka A, Hulliger M, Zangger P, Appenteng K. "Fusimotor set": new evidence for alpha-

938 independent control of gamma-motoneurones during movement in the awake cat. Brain Res 339:

939 136-140, 1985.

940 Pruszynski JA, King GL, Boisse L, Scott SH, Flanagan JR, Munoz DP. Stimulus-locked responses on 941 human arm muscles reveal a rapid neural pathway linking visual input to arm motor output. Eur J 942 Neurosci 32: 1049-1057, 2010.

943 Prut $Y$, Fetz EE. Primate spinal interneurons show pre-movement instructed delay activity. Nature 944 401: 590-594, 1999.

945 Rathelot J-A, Dum RP, Strick PL. Posterior parietal cortex contains a command apparatus for hand 946 movements. Proc Natl Acad Sci U S A 114: 4255-4260, 2017.

947 Rau G, Disselhorst-Klug C. Principles of high-spatial-resolution surface EMG (HSR-EMG): single motor 948 unit detection and application in the diagnosis of neuromuscular disorders. J Electromyogr Kinesiol 7: $949 \quad 233-239,1997$.

950 Reddi B a. J, Asrress KN, Carpenter RHS. Accuracy, Information, and Response Time in a Saccadic 951 Decision Task. J Neurophysio/ 90: 3538-3546, 2003.

952 Reddi B a. J, Carpenter RHS. The influence of urgency on decision time. Nat Neurosci 3: 827-830, 9532000.

954 Requin J, Lecas JC, Vitton N. A comparison of preparation-related neuronal activity changes in the 955 prefrontal, premotor, primary motor and posterior parietal areas of the monkey cortex: preliminary 956 results. Neurosci Lett 111: 151-156, 1990.

957 Roesch MR, Olson CR. Impact of expected reward on neuronal activity in prefrontal cortex, frontal 958 and supplementary eye fields and premotor cortex. J Neurophysiol 90: 1766-1789, 2003. 
959 Rungta SP, Basu D, Sendhilnathan N, Murthy A. Preparatory activity links frontal eye field activity 960 with small amplitude motor unit recruitment of neck muscles during gaze planning. J Neurophysiol , 961 2021. doi:10.1152/jn.00141.2021.

962 Saijo N, Murakami I, Nishida S, Gomi H. Large-field visual motion directly induces an involuntary 963 rapid manual following response. J Neurosci Off J Soc Neurosci 25: 4941-4951, 2005.

964 Schepens B, Drew T. Strategies for the integration of posture and movement during reaching in the 965 cat. J Neurophysiol 90: 3066-3086, 2003.

966 Schlag-Rey M, Amador N, Sanchez H, Schlag J. Antisaccade performance predicted by neuronal 967 activity in the supplementary eye field. Nature 390: 398-401, 1997.

968 Schmolesky MT, Wang Y, Hanes DP, Thompson KG, Leutgeb S, Schall JD, Leventhal AG. Signal 969 timing across the macaque visual system. J Neurophysiol 79: 3272-3278, 1998.

970 Selen LPJ, Shadlen MN, Wolpert DM. Deliberation in the Motor System: Reflex Gains Track Evolving 971 Evidence Leading to a Decision. J Neurosci 32: 2276-2286, 2012.

972 Sinclair C, Hammond GR. Excitatory and inhibitory processes in primary motor cortex during the 973 foreperiod of a warned reaction time task are unrelated to response expectancy. Exp Brain Res 194: 974 103-113, 2009.

975 Tanji J, Okano K, Sato KC. Neuronal activity in cortical motor areas related to ipsilateral, 976 contralateral, and bilateral digit movements of the monkey. J Neurophysiol 60: 325-343, 1988.

977 Tanji J, Shima K, Mushiake H. Concept-based behavioral planning and the lateral prefrontal cortex. 978 Trends Cogn Sci 11: 528-534, 2007.

979 Thompson KG, Bichot NP, Schall JD. Dissociation of visual discrimination from saccade programming 980 in macaque frontal eye field. J Neurophysiol 77: 1046-1050, 1997.

981 Touge T, Taylor JL, Rothwell JC. Reduced excitability of the cortico-spinal system during the warning 982 period of a reaction time task. Electroencephalogr Clin Neurophysiol 109: 489-495, 1998.

983 Zinger N, Harel R, Gabler S, Israel Z, Prut Y. Functional organization of information flow in the 984 corticospinal pathway. J Neurosci Off J Soc Neurosci 33: 1190-1197, 2013. 


\section{Figure Legends}

987

988

989

990
F1: Task and methods. A) Hand and Cursor movements: Subjects made delayed or immediate movements to the green target after the disappearance of the fixation box (go cue) to initiate a movement. The epoch between the red and green lines demarcates the delay period, whereas the epoch between the green and black line represents reaction time. B) Bar plots showing the average reaction time for delayed and immediate movements. C) Left panel. A schematic representation of a high surface density array used for recording surface electromyographic (emg) signals from the deltoid (shoulder) muscles. The reference electrode was towards the insertion point of the muscle. Middle panel. A snippet showing the propagation of signal along the direction of electrodes (1,2, 3 and 4) when analysed in differential recording mode. Right panel. Cross correlation plots showing a lag between signals recorded in differential mode with two electrodes $2.5 \mathrm{~mm}$ apart (green) or $5 \mathrm{~mm}$ apart (orange). Histogram from an example session, showing the distribution of time lags or conduction delay between electrodes that were separated by $2.5 \mathrm{~mm}$ (green) and $5.0 \mathrm{~mm}$ (orange). D) Bar plot showing the average conduction velocity estimated between electrode(s) separated by $2.5 \mathrm{~mm}$ (green) and $5.00 \mathrm{~mm}$ (orange). E) Top panel. Representative average root mean square (RMS) muscle activity from a session, aligned to the time of the go cue. Inset: shows the same activity in log scale. Bottom panel. Same muscle activity but calculated using a raster-based method. Gray markers are individual spikes and green markers are the time of movement onset. F) Density histogram showing the Pearson's correlation coefficient for data analysed using the two approaches across all sessions (Correlation between top and bottom panels in Fig $1 E$ for all sessions). 
1018 F2: Early recruitment of muscle activity based on task context. A) A representative 1019 session involving a hand movement showing the ramping up of muscle activity for delayed 1020 (left panel) and immediate movements (right panel), aligned on the go cue. Circles in olive 1021 (during delay period) and green (during reaction times), showing the onset of ramping 1022 activity across sessions for delayed and immediate movements, before and after the go cue, 1023 respectively. The filled circles show the onset in EMG activity for the corresponding example 1024 session. B) Same as A, but for cursor movements showing no modulation during delay 1025 period. C) Top panel. delayed vs immediate task: Bar plots show average activity for (i) hand 1026 (green) and (ii) cursor (grey) movements, respectively, during the delay period, prior to the 1027 go cue (Go). Bottom panel. Same as top panel but bar plots show average growth rate for (iii) 1028 hand and (iv) cursor movements during the delay period. D) Top panel. Delayed and 1029 immediate task: Bar plots show average threshold activity for (i) hand (green) and (ii) cursor 1030 (gray) movements respectively, during the reaction time (RT) following the Go cue. Bottom 1031 panel. Same as top panel but bar plots show average growth rate for (iii) hand and (iv) cursor 1032 movements during the reaction time. Error bars in black represent \pm sem, n.s. means not 1033 significant; * means $\mathrm{P}<0.05$; ** means $\mathrm{P}<0.01$; *** means $\mathrm{P}<0.001$. 
1041 F3: Early recruitment of muscle activity is spatially selective. A) A representative session 1042 showing muscle activity for the delayed and immediate hand movement task, aligned on the 1043 go cue for movements made towards (green) and away (red) from the movement field. The 1044 inset below shows the discriminability between in-MF and out-MF response for the EMG 1045 signal measured using the area under the curve (AUC) values for the session shown above. B) 1046 Same format as A but for cursor (in-MF: black and out-MF: grey) movements. C) Shown in 1047 grey are the AUC values from the ROC analysis for EMG responses in-MF and out-MF 1048 across different sessions for delayed (left) and immediate responses (right). Solid line 1049 (brown) shows the average mean value for the population. Inset. Shows the bar plot for the 1050 average increase in AUC value at the time of go cue. Triangles in olive (during the delay 1051 period) and green (during reaction times) show the direction discriminability onset for the 1052 increasing trend for AUC values across all sessions. Triangles in green (during reaction time 1053 period) show the direction discriminability onsets based on an AUC $>0.7$ that demarcates a 1054 second later phase of recruitment. The filled triangles show the onsets estimated for the 1055 corresponding session. D). Same format as C but for cursor (black) movements. n.s. means 1056 not significant; * means $\mathrm{P}<0.05$; ** means $\mathrm{P}<0.01$; *** means $\mathrm{P}<0.001$. 
1062 F4: Early recruitment of muscle activity is correlated with reaction time. A) A

1063 representative session from a hand movement session, showing ramping up of muscle activity

1064 for delayed and immediate movements, aligned on the go cue for different reaction times 1065 (RTs). Inset: Showing trial by trial scatter plot (olive) for change in activity at the time of go 1066 cue, during delay period as a function of reaction time. B) Same format as A, showing no 1067 ramping up of muscle activity for different reaction time values but for cursor movements. 1068 C). Scatter plots of muscle activity following the go cue for delayed (olive) and immediate 1069 movements (green) as a function of reaction time for the representative session shown for 1070 hand movements. D) Same format as C, but for the representative cursor session. E) Bar plot 1071 of Pearson's correlations coefficient between activity in the delay period and reaction time 1072 for delayed (olive) and immediate hand movements (green). F) Same format as E, shows bar 1073 plot for Pearson's correlations coefficient value for cursor-based movements. n.s. means not significant; * means $\mathrm{P}<0.05$; ** means $\mathrm{P}<0.01$; *** means $\mathrm{P}<0.001$. 

task. C) The bar plots show the average change in motor activity firing rate at go (first panel), 1084 onset (second panel), growth (third panel) and threshold (fourth panel) for slow (olive) and 1085 fast (green) reaction times. Each line in grey represents the data that was obtained across each 1086 session. The fifth panel represents the accumulator model that summarizes the data for 1087 delayed movements. D) same as $\mathrm{C}$ but for the immediate reach task. n.s. means not significant; * means $\mathrm{P}<0.05 ; * *$ means $\mathrm{P}<0.01 ; * * *$ means $\mathrm{P}<0.001$. 
1094 F6: Recruitment of muscle activity during cursor movements can be described by an

1095 accumulator model. A) Muscle activity during the reaction time when aligned on the go cue 1096 and movement onset in the delayed cursor task. B) same as A but for the immediate cursor 1097 task. C) The bar plots show the average change in motor activity firing rate at go (first panel), 1098 onset (second panel), growth (third panel) and threshold (fourth panel) for slow (black) and 1099 fast (gray) reaction times. Each line in grey represents the data that was obtained across each 1100 session. The fifth panel represents the accumulator model that summarizes the data for 1101 delayed movements. D) same as $\mathrm{C}$ but for the immediate cursor task. n.s. means not 1102 significant; * means $\mathrm{P}<0.05$; ** means $\mathrm{P}<0.01$; *** means $\mathrm{P}<0.001$. 
A
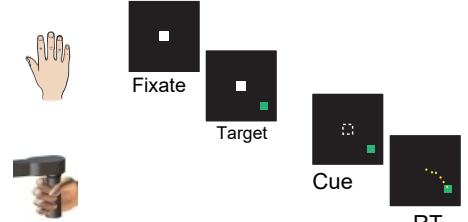

RT

Task:

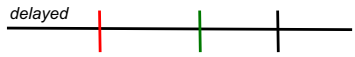

C

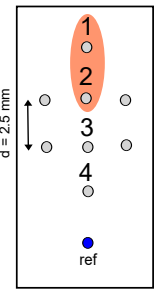

Differential recording

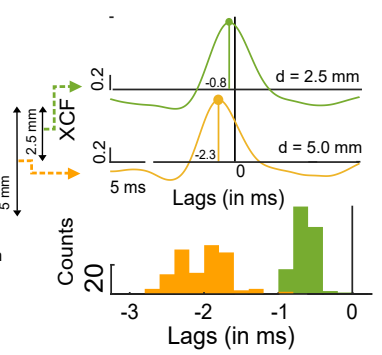

immediate

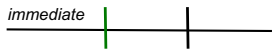

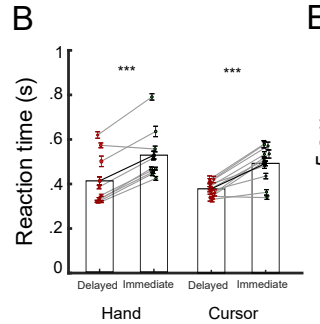

D

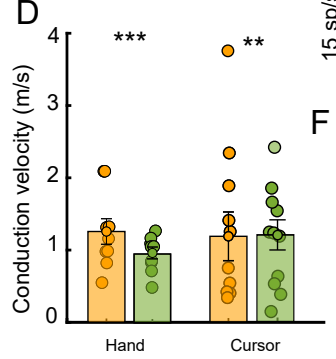

$\mathrm{F}$

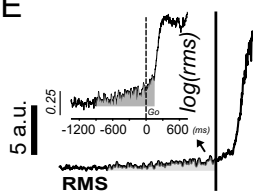

m
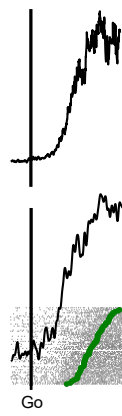

is 4 cursor hand

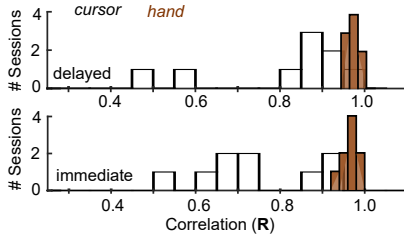


A

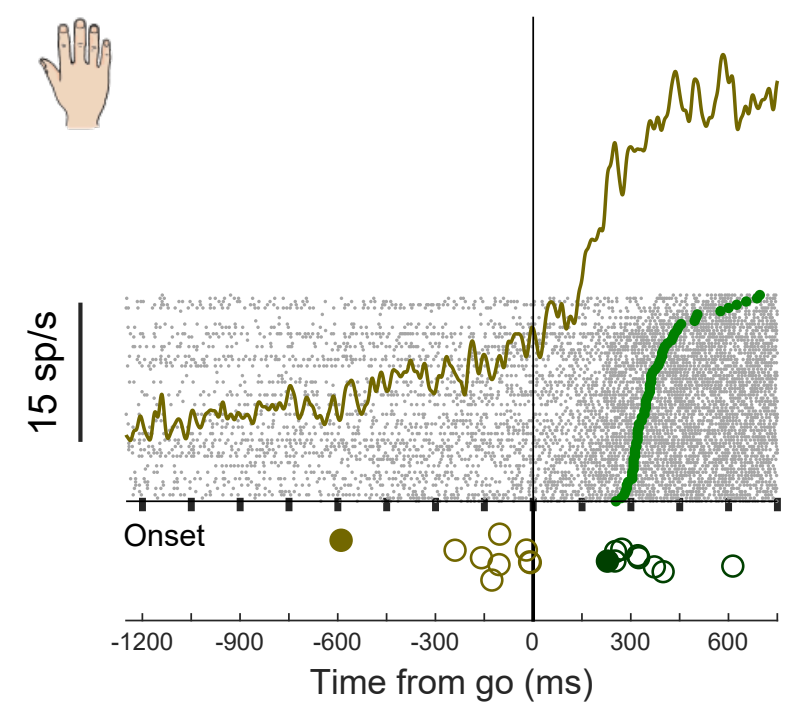

B

$\frac{\infty}{0}$
क
$\sim$
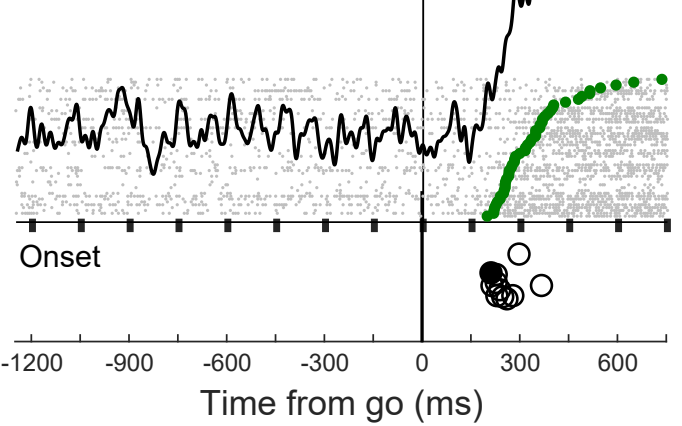

C
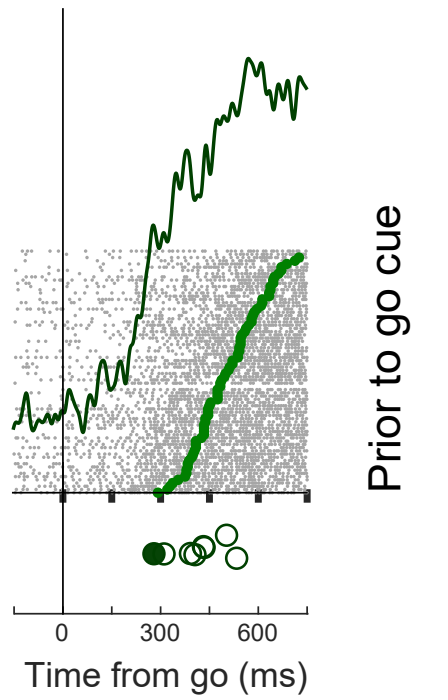

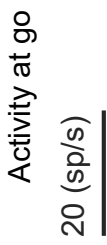

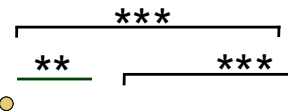

n.s.

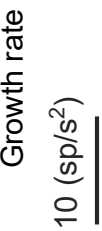
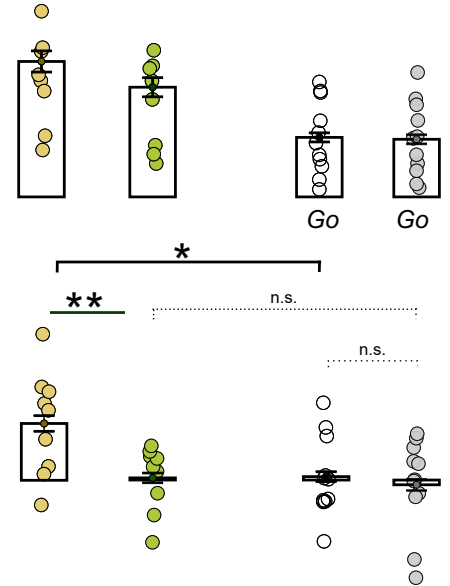

delay immediate

delay immediate

Hand

Cursor

D

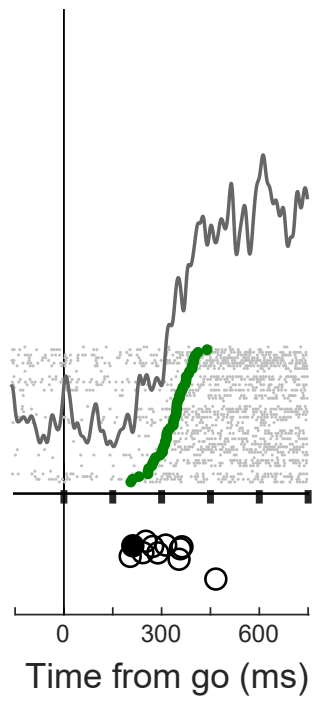

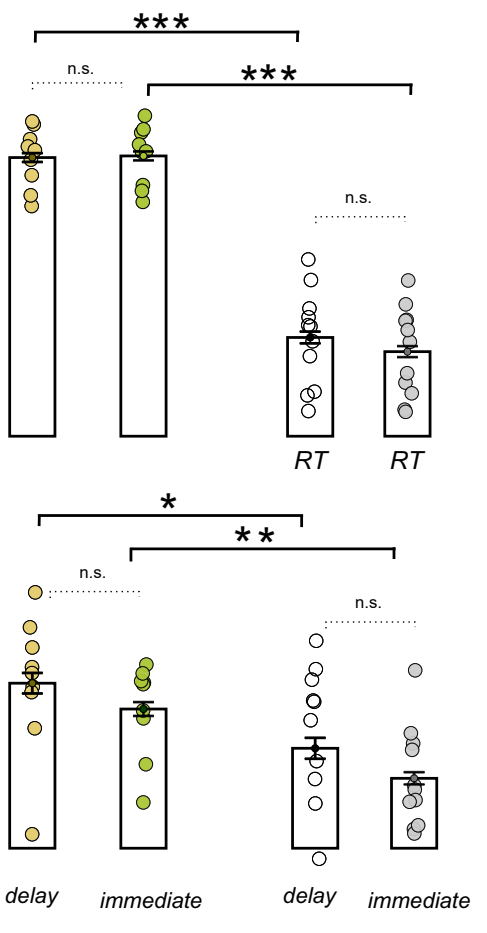

Hand 
$A$
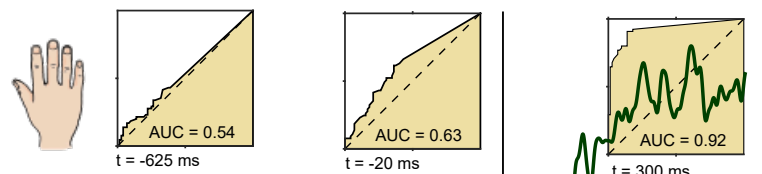

$=-625 \mathrm{~ms}$

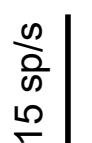

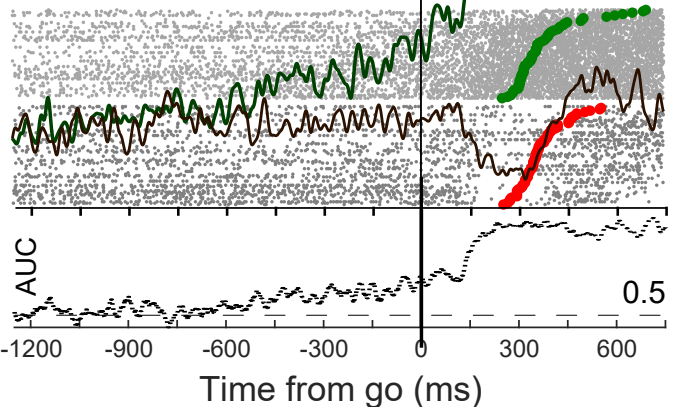

B

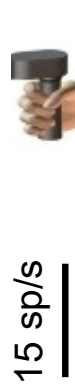

$\infty$
0
$\infty$
0
0

\ัI

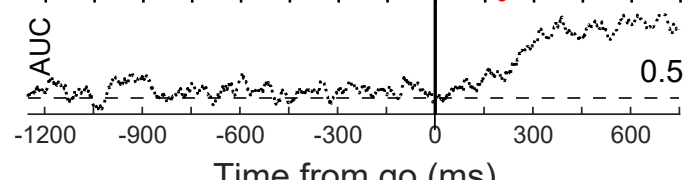

A $A$

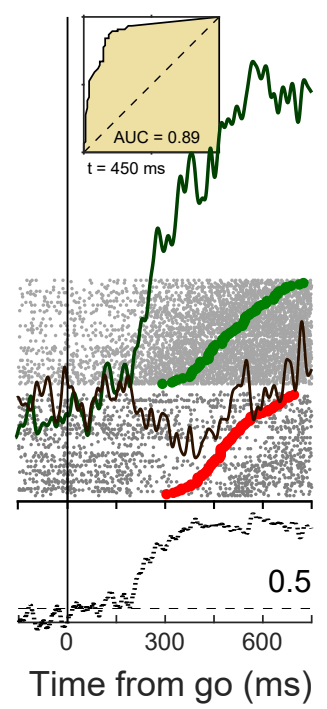

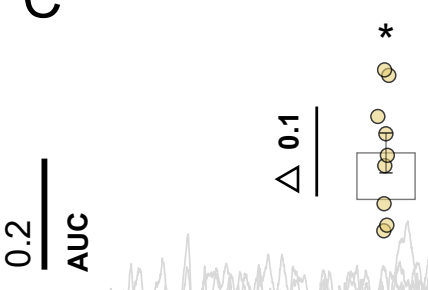
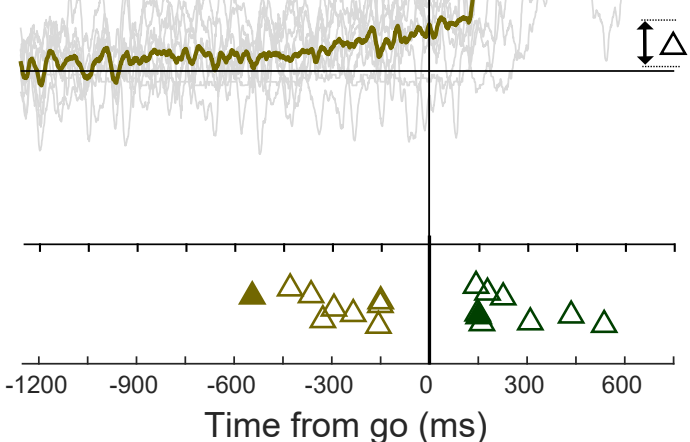

D

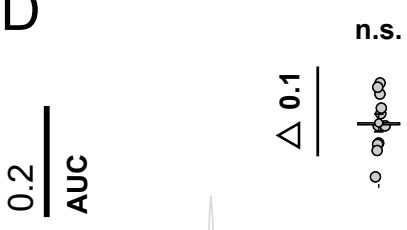

mino

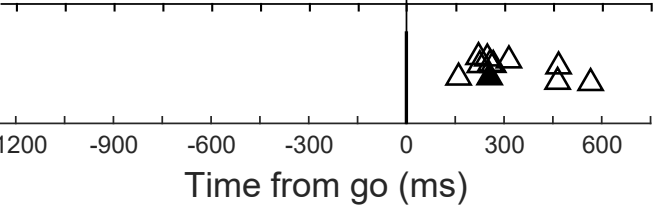

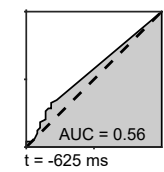

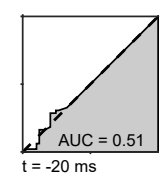

Time from go (ms)
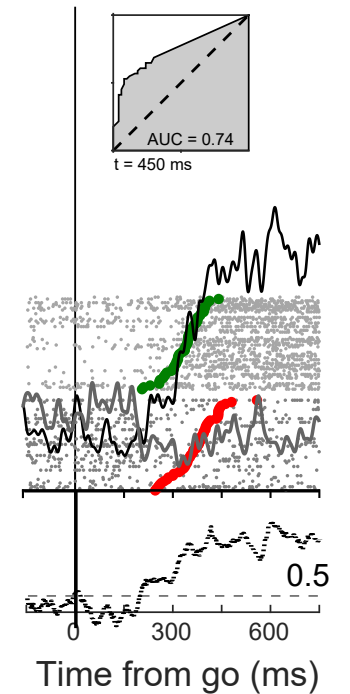

Time from go $(\mathrm{ms})$
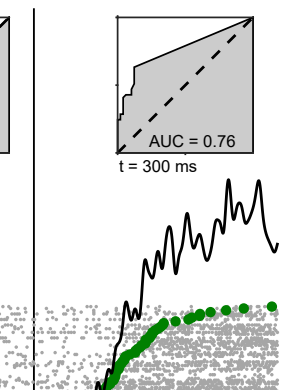

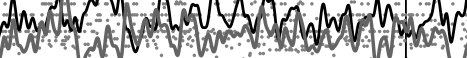

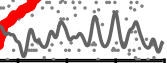

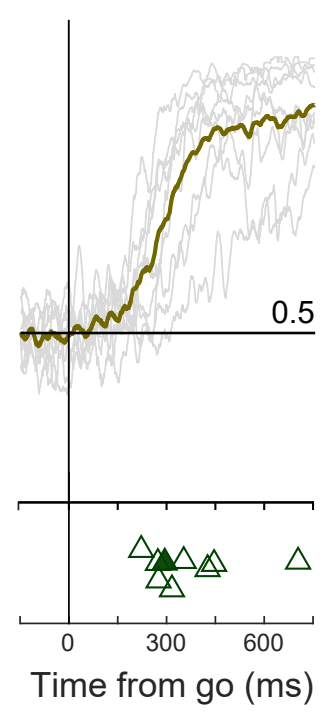

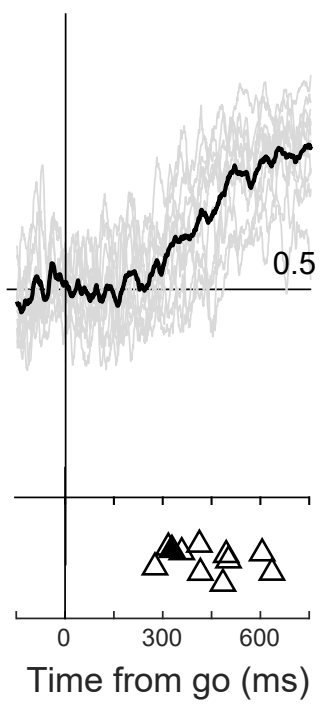



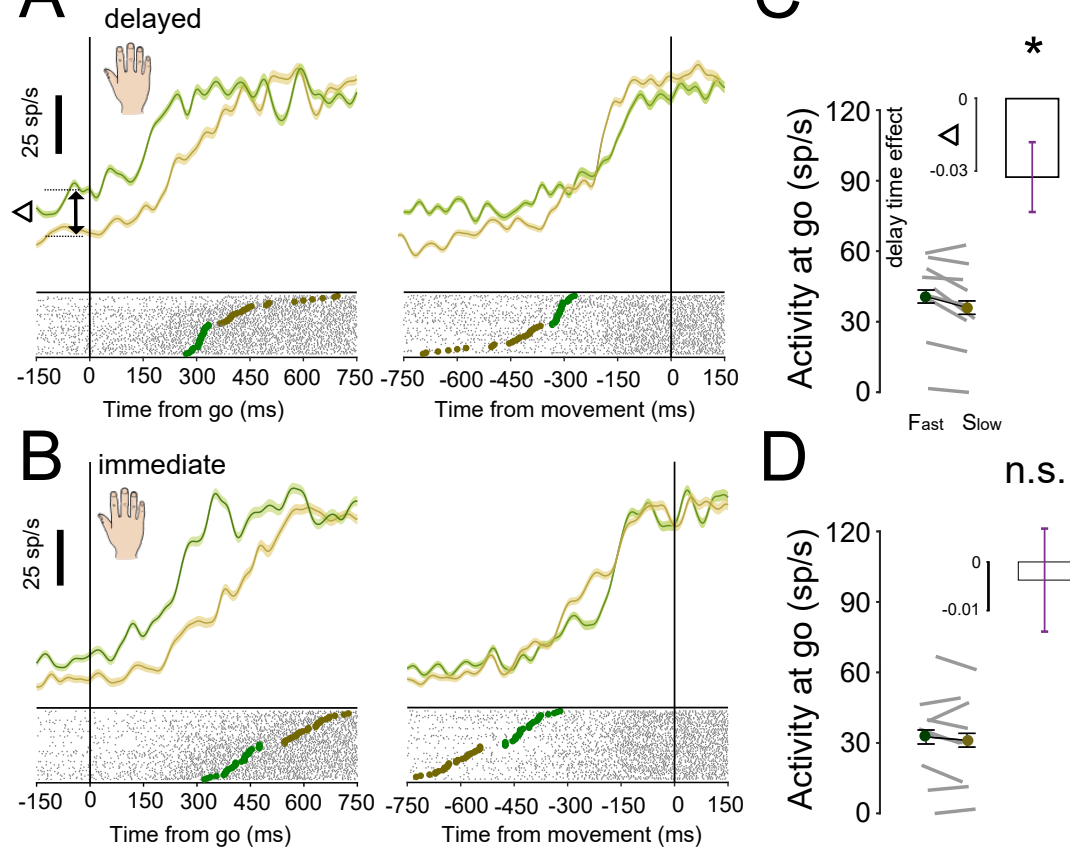

n.s.

$\overline{* * *}$

n.s.

$D$ n.s.
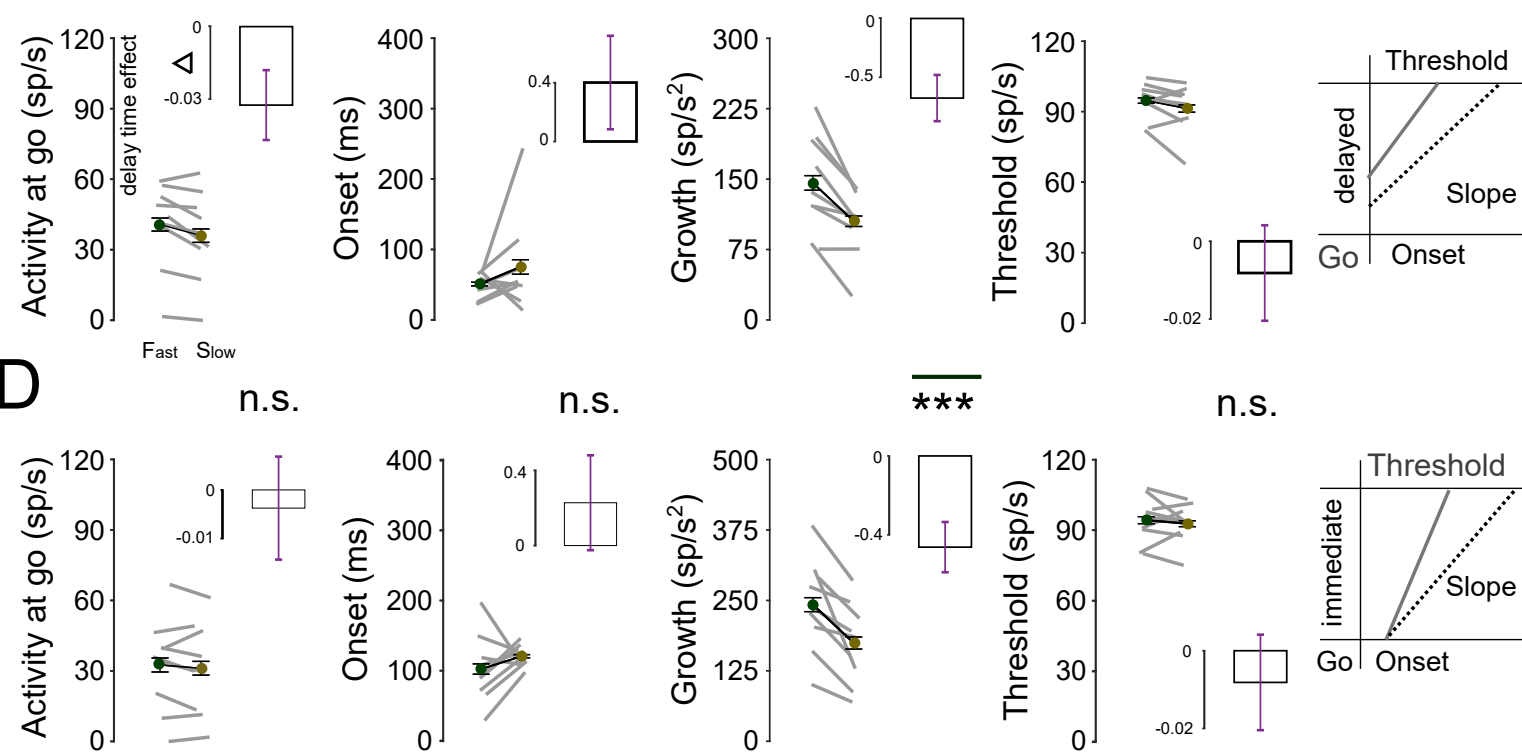

n.s.

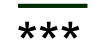

n.s.

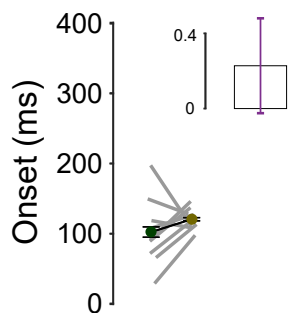

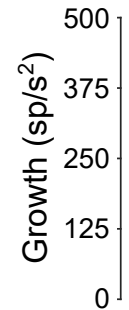
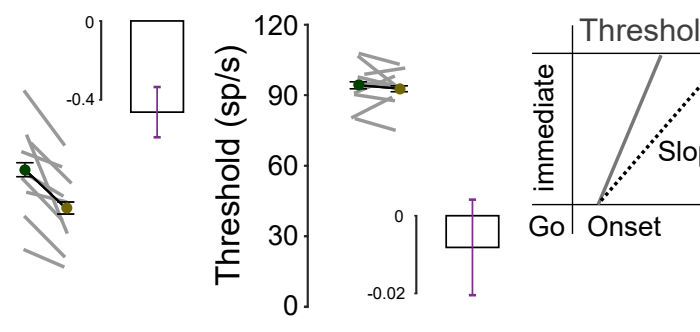


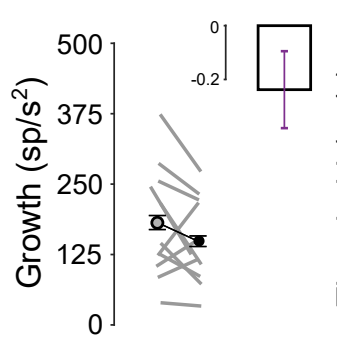

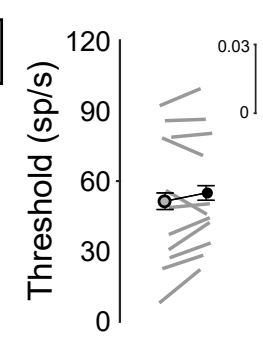<smiles>CC1CCC1</smiles>

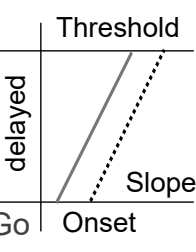

B
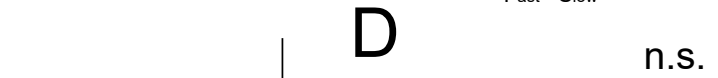

$\overline{* * *}$

n.s.

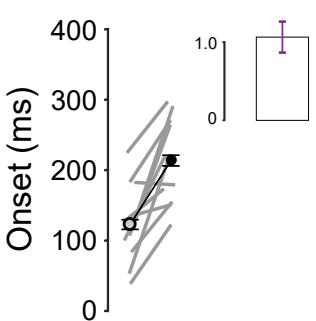

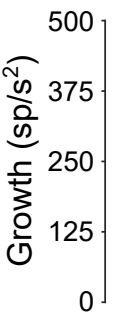
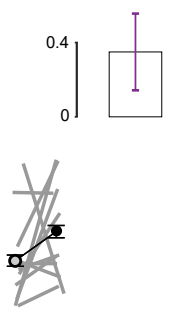

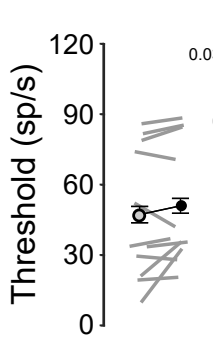

n.s.
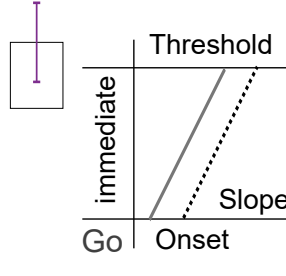

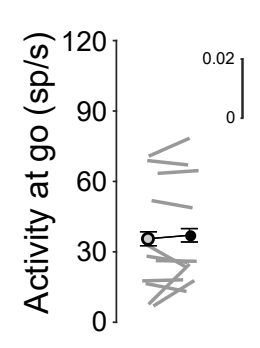

